\title{
Approaches to land snail shell bead manufacture in the Early Holocene of Malawi
}

\author{
Jennifer M. Miller ${ }^{1}$ (D) $\cdot$ Hannah M. Keller ${ }^{2}$ (D) Claire Heckel $^{3} \cdot$ Potiphar M. Kaliba $^{4} \cdot$ Jessica C. Thompson $^{2}$ (iD
}

Received: 29 August 2020 / Accepted: 5 January 2021 / Published online: 6 February 2021

(C) The Author(s) 2021

\begin{abstract}
Shell disc beads are important archeological indicators of social communication and exchange networks. There has been substantial research on ostrich eggshell (OES) beads, but little is known about the manufacture or chronology of similar beads from land snail shells (LSS). LSS beads are associated with Holocene hunter-gatherers in Africa, though direct dates are limited to the Iron Age, and there are no images or descriptions of the manufacturing sequence. Here, we combine experimental and archeological data to resolve the chronology, operational chains, and material properties of LSS bead manufacture. We then recommend and apply a modified OES production sequence to three Later Stone Age assemblages from the Kasitu Valley of northern Malawi (Hora 1, Mazinga 1, and Kadawonda 1). At these sites, LSS shows an unexpectedly high proportion of Pathway 2 manufacture (disc shaping prior to perforation), in direct contrast with known OES bead manufacture. Application of red color occurred at all stages of manufacture, albeit inconsistently. Production of finished beads involved substantial removal of material from preforms to create the final product. Finally, we report the earliest evidence for LSS bead manufacture with two directly dated preforms ( $9500 \mathrm{cal} \mathrm{BP})$, showing that the origins of LSS beadmaking do precede the Iron Age.
\end{abstract}

Keywords Chaîne opératoire · Achatinini · Symbolism · Central Africa · Eastern Africa

\section{Introduction}

Shell disc beads have been produced in Africa for at least 50,000 years (e.g., Ambrose 1998; Miller and Willoughby 2014) and have substantial implications for understanding the

Jennifer M. Miller and Jessica C. Thompson contributed equally to this work.

Jennifer M. Miller jmiller@shh.mpg.de

$\triangle$ Jessica C. Thompson jessica.thompson@yale.edu

1 Max Planck Institute for the Science of Human History, Jena, Germany

2 Department of Anthropology, Yale University, New Haven, CT, USA

3 Greensboro, NC, USA

4 Department of Museums and Monuments, Ministry of Tourism, Culture, and Wildlife, Lilongwe, Malawi emergence of symbolic behavior and the spread of ancient exchange systems (Stewart et al. 2020; Tryon 2019). The earliest disc beads were crafted from ostrich eggshell (OES), a versatile material used for personal ornaments across a wide geographic and temporal span. Archeological OES disc beads have been found as far east as China (Wei et al. 2017) and were crafted and exchanged across Africa until the modern day (Wingfield 2003). However, disc beads made from the shells of giant, terrestrial land snail shells (LSS) also appear in the archeological record across sub-Saharan Africa. They are almost identical to OES beads in shape and size and often mistaken as such (Miller et al. 2018). In spite of their ubiquity, they have received little research regarding their manufacturing pathways and chronology of appearance. Here, we report an experimental investigation of LSS breakage and shaping during the natural process of trampling versus the anthropogenic process of bead manufacture. We then use archeological data from three Later Stone Age sites in the Kasitu Valley of northern Malawi to examine the chaînes opératoires (chains of operation) of LSS disc bead manufacture and use in antiquity. Finally, we offer the earliest evidence for LSS bead manufacture in Africa, demonstrating that the roots of LSS beadmaking extend at least into the Early Holocene. 


\section{Background and previous research}

\section{The problem of land snail disc beads}

LSS beads are common elements at Holocene archeological sites but are rarely studied in detail. Miller et al. (2018) were the first to systematically compile a list of sites with LSS beads and attempt to directly date their occurrence. They found that "shell beads," many likely LSS, are described from deposits at numerous sites, but that early literature rarely specifies the type of material. Reported cases date overwhelmingly to the Iron Age or later. A few LSS beads have been reported from older contexts, but the vertical mobility of small artifacts makes their ancient status questionable. The only direct ages of LSS beads are from Magubike Rockshelter in Tanzania, where four specimens returned ages from $403 \pm$ 23 to $1732 \pm 23$ BP (Miller et al. 2018), perhaps confirming a pattern whereby LSS beads were predominately an Iron Age phenomenon.

Manufacturing techniques also remain essentially unknown. There are three possible lines of evidence to reconstruct LSS beadmaking in the past: ethnographic analogy, experimental production, and artifact description. However, there are no documented contemporary communities that practice LSS disc beadmaking in Africa, no published experimental studies, and there is a lack of archeological information on preforms or finished bead pathways at sites where confirmed LSS beads occur. Publications which do mention LSS bead preforms rarely have drawings, photographs, or intext descriptions of the artifacts. Where these exist, the drawings lack detail and assemblage-level data for understanding manufacturing processes (e.g., Phillipson 1976; Fig. 32:12, Fig. 57:12-13). Finished land snail disc beads have been illustrated in central Africa at sites such as Kalemba, Makwe, and Thandwe in Zambia (Phillipson 1976) and Malowa and Midima in Malawi (Juwayeyi 1981), but finished beads are inadequate to reconstruct manufacturing sequences. Without a systematic description or detailed images of preforms, it is impossible to study the chaînes opératoires of LSS bead manufacture from the available archeological literature.

Another hurdle LSS researchers face is that culturally modified LSS may not be recognized or collected during excavation. Shell fragments from large terrestrial mollusks are common at African archeological sites, but their presence may result from a natural accumulation or food processing debris, and not necessarily bead production. Recovery methods for small finds have historically been variable across sites, making it difficult to have confidence that the entire potential spectrum of manufacturing debris has been curated and is available for study. Therefore, even where analysis of original assemblages is possible, they may be incomplete or biased against objects that are of a particular size and/or shape.

\section{Land snail shell versus ostrich eggshell}

Understanding the manufacture and use of shell disc beads goes beyond understanding technological decisions, as shell beads have played an important role in social networks, both today and in the ancient past. OES beads in particular are a favored item in !hxaro, an intragroup gift exchange system practiced among hunter-gatherers in southern Africa (Marshall 1976; Smith and Lee 1997). In fact, the word !hxaro is used synonymously in some communities as the term for OES beadwork (Mitchell 2002: 36) A valued !hxaro item may travel as far as several hundred kilometers in two years, being gifted several times along the way (Lee 1984: 99). Recent research by Stewart et al. (2020) used isotope provenience of OES beads to show long-distance travel in the past, suggesting that this practice of gifting OES beads may have begun more than 30 thousand years ago.

The manufacture of archeological OES beads has been studied in some detail, and a protocol exists for the description of their preforms. The OES bead manufacturing sequence was codified by Kandel and Conard (2005), then later refined by Orton (2008). The coding system is based on observations of archeological specimens and modern ethnographic research; today, it is widely used to describe OES preforms from African archeological assemblages. Orton (2008) divides the chaîne opératoire of OES beadmaking into two distinct pathways. Both pathways begin by procuring fragments of OES that are a suitable size for beadmaking, which may involve snapping OES fragments into square or triangular pieces a few centimeters in diameter. Pathway 1 (PW1) then proceeds with creating a perforation through the shell, before shaping the outer rim. With this method, many perforated blanks can be strung together, trimmed by chipping away rough edges with a piece of horn, and then rubbing the blanks with a soft stone to achieve the final uniform shaping. In contrast, Pathway 2 (PW2) preforms have their outer rim shaped into a disc first; then, an aperture is drilled. With this method, it is not possible to do the rough shaping of multiple beads at once, so Pathway 1 appears to be the more efficient method of producing quantities of beads with similar shapes and sizes. This is reflected in the ethnographic and archeological records where instances of PW1 manufacture (Bleek 1928; Dunn 1931; Hahn et al. 1966; Lee 1984; Miller 2019; Silberbauer 1981; Van der Post and Taylor 1985; Wingfield 2003) far outweigh PW2 (Stow 1905).

Thorough descriptions and photographs of each stage of manufacture offer a common framework within which researchers can systematically categorize and compare pathways over both space and time. In human societies, technological chains where different pathways lead to similar end results may be informative about processes of cultural transmission (Jordan 2015). Thus, approaches to artifact manufacture can offer higher-level interpretations of ancient social 
relationships. However, the performance attributes of different raw materials can also have a strong influence on artifact manufacture, because they may make some approaches more or less efficient than others. In the context of objects commonly found at Stone Age sites, comparing how raw materials influence the manufacturing processes and final products has mainly been done for stone tools (Braun et al. 2009; Eren et al. 2011; Eren et al. 2014) and only in one case for disc beads (Langley 2018).

Intuitively, there are numerous similarities between OES and LSS that may have prompted ancient peoples to use the different materials in similar ways. Both types of shells have a wide terrestrial distribution throughout Africa and can be collected with little to no skill or danger. Ostriches can be dangerous, but San and Bakgalagadi people of Botswana have been known ethnographically to contrive ways to get the birds to leave the nests long enough to collect one or more eggs (Hitchcock 2012). It is conceivable that a strategy in the past for bead manufacture might also have been to collect fragments of eggs from nests abandoned after a clutch of eggs had hatched, as a single ostrich nest could contain more than 20 eggs (Jarvis et al. 1985: 442). Another similarity between the two raw materials is that both types of shells have edible contents, and while some land snails are quite small, giant terrestrial species of the tribe Achatinini are documented food sources in West Africa (Mead 1961; Otchoumou et al. 2010). OES and LSS can also both be used as containers. Although a giant snail shell cannot hold as much fluid as an ostrich egg, they have been used as salt containers in northeastern Tanzania (Walz 2017: 94). Both types of shells are durable and can achieve thickness suitable for drilling, although LSS thickness varies more according to the size of the individual and anatomical location of the fragment.

These similarities between OES and LSS may also extend to their bead manufacturing sequences, but this can only be confirmed with experimental and archeological observations. Here, we draw our initial framework from research on OES, which does have ethnograhically documented chains of manufacture, to guide experimental work with LSS. We then examine the manufacturing debris and finished products from three recently excavated sites in Malawi at which all LSS fragments larger than $3 \mathrm{~mm}$ were recovered and available for analysis.

\section{Materials and methods}

\section{Experimental methods}

We acquired an assemblage of modern shells through the Florida State Eradication Program as a proxy for the archeological LSS from Malawi. The project's website classifies the Floridian pests as invasive giant African snails, which is an unofficial taxon encompassing large terrestrial genera in the tribe Achatinini. Genera that occur in Malawi include Lissachatina (sometimes listed as Achatina), Bequaertina, and Bruggenina (Van Bruggen 2008; MolluscaBASE Creative Commons Attribution 4.0 International Public License). The archeological literature frequently describes terrestrial snail remains from central Africa, including beads, as derived from Achatina (Clark 1956; Phillipson 1976). However, Miller et al. (2018) caution that modern distributions of land snails may be different from past distributions, citing complete shells from the archeological site of Magubike in southern Tanzania that are a closer match to Burtoa nilotica. Furthermore, there have been a number of recent taxonomic revisions within Achatinini, and several genera contain multiple species (Mead 2004; Van Bruggen 2008). Thus, it is unlikely that all archeological specimens are from the same species of land snail, nor that all or even most of these are Achatina. Complete specimens and clusters of fragments from the Holocene archeological deposits at two of the archeological sites described here are illustrated in Fig. 1. A summary of all experimental aims, methods, and outcomes is provided in Table 1 .

\section{Breakage}

Evidence of drilling is a reliable indicator of bead manufacture, but in the absence of an aperture, it can be difficult to distinguish natural from intentional shaping. This becomes particularly problematic in PW2 manufacture, when earlystage preforms may be semirounded discs with no trace of an aperture. Because the first stage of any disc beadmaking is fragmentation, we attempted to fragment the shells using four main approaches: crushing under body weight, breaking by hand, breaking with percussion, and crushing using a hammerstone and anvil.

To simulate natural breakage from trampling, we crushed six shells by stepping on them. We laid three on a hard linoleum floor and three on the grass to simulate a softer surface. We then simulated breakage by human manipulation without the specific intent of creating bead preforms, by breaking one specimen apart by hand. An internet search for cooking techniques of giant land snails shows that they can be boiled whole or extracted by pressing a rod into the aperture to skewer and extract the snail body. We replicated this extraction technique with a metal chisel and found that it was an effective way to fracture the shell. Similar examples of fragmented shells with a single hole have been found archeologically in clusters at Hora 1, suggesting possible human intervention (Fig. 1). Finally, we attempted to fragment the body whorl specifically to create pieces suitable for preform manufacture. We used a ball-peen hammer and metal chisel, then broke the shell with a hammerstone and anvil. 

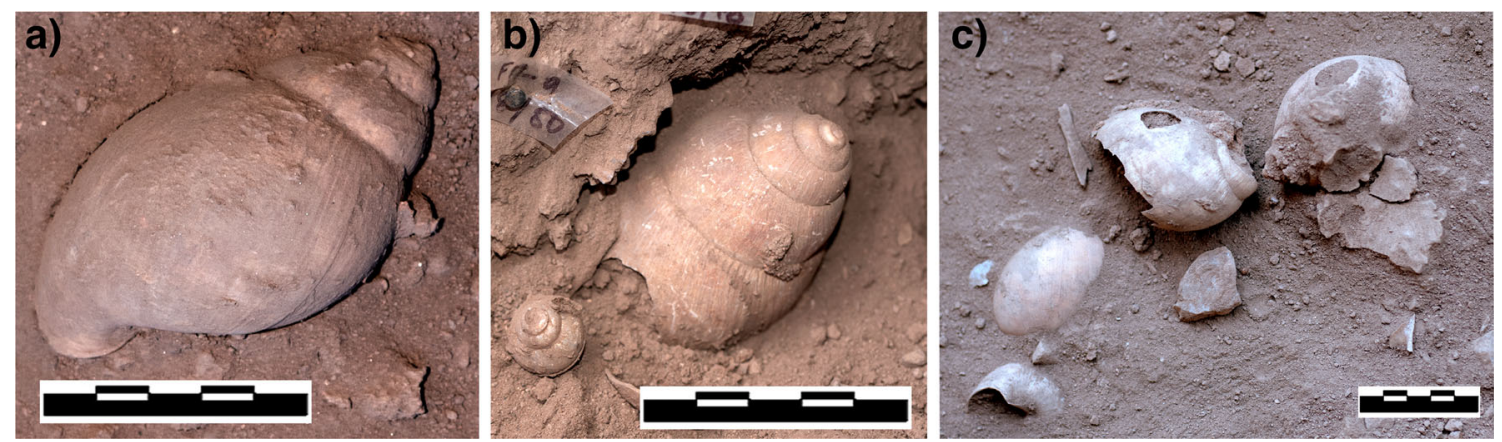

Fig. 1 Examples of intact giant land snails in situ at the (a) HOR-1 and (b) MAZ-1 sites in the Kasitu Valley of Malawi. Both are from Holocene layers. Panel (c) shows clusters of shells with holes at similar parts of the anatomy, also been found at HOR-1

\section{Shaping}

In the absence of ethnographic observations, we made our goal to replicate the shape of unambiguous archeological LSS preforms, identified as such by being fully rounded on all sides, or where an aperture hole had been started or had penetrated completely. Since part of our goal is to identify LSS preforms in archeological assemblages that may not have an aperture, we attempted to replicate the shaping of those specimens which had only outer shaping. Using fragments from the breakage experiments, we selected pieces $1-3 \mathrm{~cm}$ in diameter to be further shaped into round discs.

In OES production sequences, initial shaping produces polygonal fragments of shell, which can be easily and accurately created with pressure between the forefingers and thumbs (Wingfield 2003: 56-57). We attempted an initial rough shaping of LSS into a polygon in the standard way documented for OES, by holding a fragment and snapping off obtuse corners. When this failed, we then attempted hard hammer percussion, rubbing/grinding with a coarse substrate, and finally pressure flaking. For simplicity, we used a thick fragment of LSS as a pressure flaker. We tried pressure flaking with the outer shell surface facing towards, and then away, from the flaker.

\section{Drilling}

Once an aperture exists, there is no ambiguity about natural versus intentional modification. Identification of early-stage preforms may therefore be aided by knowing the threshold at which a fragment is too thin to stand up to the rigors of drilling, which is the next stage of manufacture. To test whether a minimum thickness threshold could be established, we applied pressure to 30 shell fragments of varying thickness and noted the results. Shell thickness was measured with digital calipers and recorded in millimeters to two decimal places. In cases where shell thickness was variable, an average was calculated from the highest and lowest measurements. We then applied force to the shells using a bamboo skewer as they sat on a digital scale and recorded the weight at breakage to the nearest hundred grams.

\section{Pigments}

Many beads/preforms bear red residue, which is often presumed to be intentionally applied ochre. This hypothesis typically goes untested, though some recent publications suggest that this tide is turning (see Collins et al. 2020; Velliky et al. 2021). For our study, we used a portable X-ray Fluorescence (pXRF) device to test whether the quantity of iron recorded can be reliably used to indicate the presence of ochre. We fragmented two modern land snail shells with a hammerstone. We then selected thirty fragments of a size and thickness representative of the archeological specimens and scanned them with the pXRF device. We used an Olympus Vanta VMR pXRF, which includes an Rh node, 4-W X-ray tube, and a large area $\left(40 \mathrm{~mm}^{2}\right)$ silicon drift detector (Frahm and Tryon 2018). Low-noise signal processing electronics allow for high count rates (over 100,000 counts per second) and high resolution $(<140 \mathrm{eV})$. There is no vacuum involved in taking measurements. Although data on other elements were collected in each measurement, only the results of $\mathrm{Fe}$ are considered here. Specimens were placed on the aperture and covered with a shield, and scanned twice, once on each side, for each of three rounds. Round one consisted of unmodified LSS with no pigment applied. One of us then mixed "burnt sienna" ochre purchased from Kremer Pigmente with beef tallow, applied it to both surfaces of LSS using a cotton tip, and then scanned them again. Subsequently, we immersed the specimens in clean tap water and gently rubbed them with a cotton tip before scanning a final time.

\section{Archeological samples}

The most reliable way to reconstruct manufacturing sequences that were actually used in the past is with the aid of ancient bead preforms. These are artifacts that were broken, lost, or otherwise abandoned during the process of bead formation, having never reached the end stage. However, not all preforms are equally useful for reconstructing manufacture. Those from early manufacturing stages carry the most information, and once a specimen has reached the point where there is a 
Table 1 Summary of the experiments employed in this study, their aims, primary methods, and outcomes

\begin{tabular}{|c|c|c|c|c|}
\hline & Question & Steps & Outcome & Implication \\
\hline LSS breakage & $\begin{array}{l}\text { How did people obtain } \\
\text { fragments for LSS preforms? } \\
\text { Can we distinguish naturally } \\
\text { vs. intentionally broken LSS } \\
\text { that may be products of } \\
\text { beadmaking activity? }\end{array}$ & $\begin{array}{l}\text { Break modern LSS using } \\
\text { different methods } \\
\text { (trampling, by hand, metal } \\
\text { hammer, and stone hammer } \\
\text { and anvil) and examine } \\
\text { resulting fragments for } \\
\text { distinguishing features. }\end{array}$ & $\begin{array}{l}\text { Stone hammer and anvil was } \\
\text { the most effective method } \\
\text { for breaking LSS, while } \\
\text { hammer and chisel were the } \\
\text { least. All breakage produced } \\
\text { similar fragments. }\end{array}$ & $\begin{array}{l}\text { The hammer and anvil method } \\
\text { is the easiest way to produce } \\
\text { fragments, but all successful } \\
\text { breakage produced } \\
\text { fragments with similar } \\
\text { appearances (linear, } \\
\text { rectangular, triangular, and } \\
\text { semirounded). We found no } \\
\text { way to distinguish } \\
\text { intentionally broken from } \\
\text { naturally broken LSS. }\end{array}$ \\
\hline LSS shaping & $\begin{array}{l}\text { Which shaping technique(s) } \\
\text { leaves traces similar to those } \\
\text { on the archeological LSS } \\
\text { preforms? Can these data } \\
\text { (along with breakage results) } \\
\text { be used to identify early stage } \\
\text { preforms? }\end{array}$ & $\begin{array}{l}\text { Use suitable fragments from } \\
\text { breakage experiment, try } \\
\text { different shaping techniques } \\
\text { (snapping by hand, hard } \\
\text { hammer percussion, indirect } \\
\text { percussion, pressure flaking, } \\
\text { and grinding) and compare } \\
\text { results. }\end{array}$ & $\begin{array}{l}\text { Pressure flaking produced } \\
\text { results similar to the } \\
\text { archeological LSS preforms. } \\
\text { Most other techniques were } \\
\text { unsuccessful at shaping. }\end{array}$ & $\begin{array}{l}\text { Archeological preforms were } \\
\text { almost certainly shaped by } \\
\text { pressure flaking, with later } \\
\text { finer shaping by grinding. } \\
\text { Natural breakage produced } \\
\text { some semirounded } \\
\text { fragments, but none with } \\
\text { more than 50\% rounded. An } \\
\text { early-stage LSS preform } \\
\text { should bear signs of pressure } \\
\text { flaking and at least } 50 \% \\
\text { shaping. }\end{array}$ \\
\hline LSS drilling & $\begin{array}{l}\text { Is there a minimum thickness for } \\
\text { LSS to become a bead? How } \\
\text { thick should a shell be to } \\
\text { survive drilling pressure? }\end{array}$ & $\begin{array}{l}\text { Apply pressure with a pointed } \\
\text { implement to LSS fragments } \\
\text { of varying thickness. Record } \\
\text { the force applied with a } \\
\text { digital scale. We used } \\
1360 \mathrm{~g} \text { as the baseline value } \\
\text { for drilling force. }\end{array}$ & $\begin{array}{l}\text { Out of } 30 \text { fragments, all those }< \\
0.50 \mathrm{~mm} \text { thick }(n=12) \text { broke } \\
\text { before reaching } 1360 \mathrm{~g} \text { of } \\
\text { force. }\end{array}$ & $\begin{array}{l}\text { Shells thinner than } 0.50 \mathrm{~mm} \\
\text { are unlikely to survive the } \\
\text { drilling process and should } \\
\text { not be considered as } \\
\text { preforms. }\end{array}$ \\
\hline Ochre and $\mathrm{pXRF}$ & $\begin{array}{l}\text { Is there a statistically significant } \\
\text { difference between the } \\
\text { quantities of iron detectable } \\
\text { with pXRF for land snail shell } \\
\text { fragments with no ochre, } \\
\text { ochre applied with a binder, } \\
\text { and after washing? }\end{array}$ & $\begin{array}{l}\text { Apply ochre to fragments of } \\
\text { LSS of similar size and } \\
\text { thickness to archeological } \\
\text { specimens, using a standard } \\
\text { tallow binder. Scan each } \\
\text { specimen } 6 \text { times: once } \\
\text { prior to ochre application, } \\
\text { once with ochre, and once } \\
\text { after the ochre is removed } \\
\text { with water (one scan per } \\
\text { experiment per side). }\end{array}$ & $\begin{array}{l}\text { A significant difference in the } \\
\text { Fe Wt } \% \text { was detected } \\
\text { between each scan. }\end{array}$ & $\begin{array}{l}\text { pXRF technology can detect } \\
\text { increases in iron as red earth } \\
\text { pigments are applied with a } \\
\text { binder to LSS, and Fe levels } \\
\text { remain higher than baseline } \\
\text { even after washing. }\end{array}$ \\
\hline
\end{tabular}

complete perforation and a rounded disc, signs of the pathway have been removed. These late-stage preforms and beads are still important artifacts, but no longer bear the evidence required to discern the order of aperture drilling and rim shaping. Therefore, in order to use archeological data to reconstruct ancient techniques, an assemblage with early-stage preforms is required. This assemblage should derive from an archeological site where there has been full recovery of LSS in addition to completed artifacts, so that the apparently unmodified shell can be systematically sorted for the presence of preforms. Three sites in the Kasitu Valley of northern Malawi, Hora $1\left(11^{\circ} 39^{\prime} 30.75^{\prime \prime} \mathrm{S}, 33^{\circ} 38^{\prime} 38.08^{\prime \prime} \mathrm{E}\right)$, Mazinga $1\left(11^{\circ} 43^{\prime}\right.$ $\left.21.11^{\prime \prime} \mathrm{S}, 33^{\circ} 42^{\prime} 7.43^{\prime \prime} \mathrm{E}\right)$, and Kadawonda 1 (11 ${ }^{\circ} 41^{\prime} 59.03^{\prime \prime} \mathrm{S}$, $33^{\circ} 45^{\prime} 23.00^{\prime \prime} \mathrm{E}$ ), meet these criteria (Fig. 2).

\section{Site descriptions}

The Hora 1 (HOR-1) site was first excavated in 1950 by J. Desmond Clark (1956). The site is best known for its recovery of two flexed adult human burials, dated to ca. $8200 \mathrm{cal} \mathrm{BP}$, and which produced the oldest ancient DNA from sub-Saharan Africa (Morris and Ribot 2006; Skoglund et al. 2017). The majority of associated finds have been lost, if in fact they were ever originally curated. Highly selective recovery during the original excavation is likely, given the dominant practices at the time and Clark's (1956: 105) statement on recovery of the faunal assemblage that emphasizes complete, identifiable elements. The use of a sieve is also uncertain; from archival photographs, the volume of sediment appears to be $\sim 12 \mathrm{~m}^{3}$, and yet Clark (1956: 106) reports the 


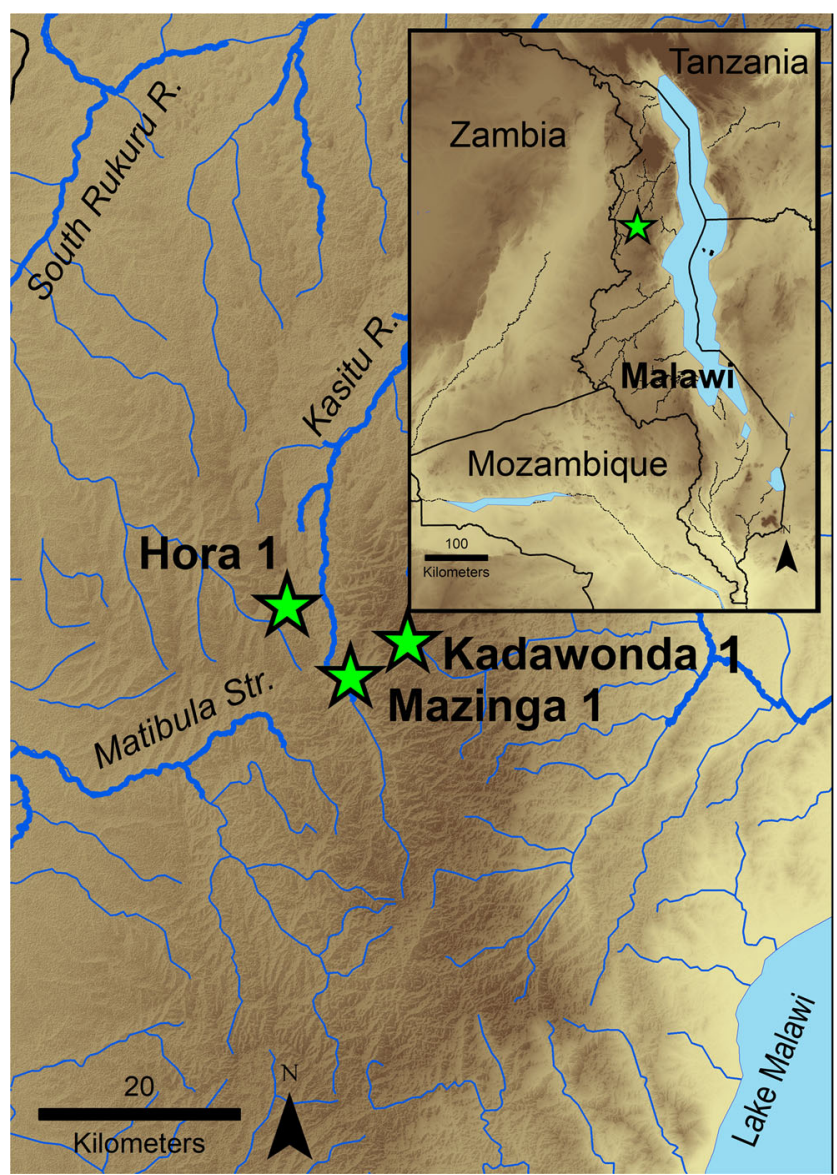

Fig. 2 Location of the three sites from which the archeological LSS bead assemblages are derived. All coordinates provided in the text are exact site coordinates taken by one of us (JCT) with handheld GPS

recovery of only three disc beads. He specifies that these were “... made probably from Achatina sp. of which quite a number of shells were found in the deposits of the shelter." For reference, our excavations that used a $1 \mathrm{~mm}$ sieve at the site have recovered 60 complete and 40 broken disc beads (collectively made from LSS, OES, and aquatic shell) from $\sim 7 \mathrm{~m}^{3}$. This is 57 times the concentration of beads per volume of excavated sediment in comparison to the original Clark excavations, strongly suggesting that either no sieve was used, the sieve aperture was too large to recover beads, or beads were selectively sampled rather than collected in full.

We renewed archeological excavations at HOR-1 between 2016 and 2019, reaching bedrock and completing excavations under the Malawi Ancient Lifeways and Peoples Project (MALAPP). The project has a primary goal of providing cultural and chronological context for HOR-1 and other sites in and near the Kasitu Valley through finescaled excavations with recovery of all artifacts and ecofacts $>1 \mathrm{~mm}$. The Mazinga 1 (MAZ-1) and Kadawonda 1 (KAD-1) sites were discovered during a survey in 2016, with MAZ-1 excavated from 2017 to 2019 and KAD-1 only in 2017. Work is ongoing at both. An additional complete LSS bead was recovered from Late Holocene deposits at the Hora 5 (HOR-5) site, at $11^{\circ} 39^{\prime} 24.57^{\prime \prime} \mathrm{S}$, $33^{\circ} 38^{\prime} 21.56^{\prime \prime} \mathrm{E}$, but as it is the only specimen from the site, it is not included in the analyses here. Archeological pigments were recovered at both HOR-1 and MAZ-1, and basic measurements of their iron content are included in our analysis below. All sites are granitic rock shelters with shallow overhangs that preserve large quantities of bone, shell, charcoal, and stone tools in a powdery sediment. Excavation and recording methods at all sites were identical.

\section{Recovery protocols}

Excavations took place within a grid of $50 \times 50 \mathrm{~cm}$ squares, with a maximum spit thickness of $5 \mathrm{~cm}$. If natural layers or individual features could be discerned, they were removed separately. All artifacts or ecofacts identified during excavation and at least $1 \mathrm{~cm}$ in size were piece-plotted with a total station and given a unique identifier using a barcoded recording system (Oestmo and Marean 2014). Beads were always plotted if they were discovered in situ. Spatial coordinate data were also collected for the extents of excavation contexts, large unmodified rocks, shelter walls, and specialist samples. All sediments were washed at the field lab through nested $1 \mathrm{~mm}$ and $3 \mathrm{~mm}$ wet sieves, and the $3 \mathrm{~mm}$ fraction was sorted into categories (lithic, bone, shell, charcoal, etc.). Only lithic, bone, and metal objects were retained during $1 \mathrm{~mm}$ sorting, because the extremely high fragmentation of materials such as shell and charcoal exponentially increased sorting effort without providing specimens that were large enough to be informative either about taxon (in the case of charcoal) or as anthropogenic modifications (in the case of shell). However, broken beads with an aperture occasionally slipped sideways through the $3 \mathrm{~mm}$ fraction and into the $1 \mathrm{~mm}$ fraction, and these were retained. Of the 245 LSS specimens reported here (the number 245 includes the one from HOR-5, which is not included in any of the analyses), 232 were recovered in one of the two sieves, rather than piece-plotted.

\section{Sorting, data collection, and analysis}

All shell recovered in the $3 \mathrm{~mm}$ fraction from the sites was sorted to recover any potential beads, preforms, bead manufacturing debris, or other modified shell fragments. The 
same individual sorted all the 2016-2018 assemblages, and two different individuals worked together to sort the 2019 assemblages. Sorting was done under a bright light and with the aid of a 10-20x hand lens. Shell fragments recovered in the sieve were extracted for further investigation if they had any evidence of chipping, striations, color, or drilling or were round in shape. These debris candidates and all beads were then photographed from both sides with a DinoLite digital microscope. Specimen photographs were imprinted with both the provenience information of the specimen and the unique specimen number. The original specimens were then examined by one of us (JM) to determine the raw material type, pathway, and other manufacturing notes.

The total HOR-1 LSS bead/bead manufacturing debris assemblage comprises 73 beads, 91 preforms, and 24 probable preforms, from a total excavated volume of $7.1 \mathrm{~m}^{3}$. The Mazinga 1 LSS bead assemblage to date, and reported here, comprises 22 beads, 13 preforms, and 6 probable preforms from a total excavated volume of $4.3 \mathrm{~m}^{3}$. The Kadawonda 1 assemblage comprises 12 beads and 3 preforms from $1.1 \mathrm{~m}^{3}$, for a total assemblage of 244 from the three sites. Unless otherwise noted, probable preforms are included in the analyses below as preforms.

Raw material and production stage were identified using a combination of visual inspection and low-powered microscopy. Using the protocols in Miller et al. (2018), experimental samples of shells were compared to the archeological beads to identify shell microstructures. Those with the alternating cross-lamellar layers were categorized as LSS. Bead manufacture was recorded using the sequence by Orton (2008). CH then photographed the more complete specimens using a standard backdrop and conducted the morphometric analysis. Bead thickness was measured using digital calipers to the nearest $0.10 \mathrm{~mm}$.

Morphometric analysis of LSS bead preforms and beads was conducted in the freeware program Tomato Analyzer following the protocol for the analysis of archeological beads established by Heckel (2016). This approach provides a more detailed quantitative assessment of disc-bead preforms and demonstrates the nuanced differences in production stages. We use it here to quantify the morphological changes in bead preforms over the course of the production process for the samples that present adequately large sample sizes (Fig. 3). Given the small sample sizes, normal distribution and homogeneity of variance were first evaluated using the Kolmogorov-Smirnov test for normal distribution and Levene's test for homogeneity of variance.

Nearly half $(N=103)$ of the beads and preforms exhibited red coloration visible with 10-40x microscopy (Supplementary Fig. 1). This red "residue" may have been an earth pigment such as ochre applied to the bead after preform manufacture, after bead manufacture, or during the process of wearing close to skin with adhering pigment (Collins et al. 2020). Conversely, it may be red staining transferred from the surrounding matrix during deposition, or some other natural process. Because iron is incorporated into the shells of living species of giant land snails (Ademolu et al. 2016), traces of iron are expected even among the LSS with no residue or sediment. However, anthropogenic application of pigments to the beads at a given stage in their production or wear through either deliberate or accidental processes should create an observable increase in the iron content compared to unmodified LSS from the same depositional contexts.

To examine the amount of iron present on beads and preforms, HMK and JCT first recorded the percentage of the specimen covered by adhering sediment and/or red residue in $10 \%$ increments. All red residues on the surface of the specimens were treated as a "potential" pigment signature. We coded the quantity of residues as "none," "trace" (some residue, but $\leq 20 \%$ of any surface or the aperture covered), and "substantial" ( $\geq 30 \%$ of at least one surface or the aperture covered). To identify the point in the manufacturing sequence at which pigment was introduced to the LSS beads, we then collected data on the elemental composition of preforms, beads, and unmodified LSS. HMK scanned 92 beads, 116 preforms, and 539 unmodified shells with the pXRF. Scanning of an unmodified shell was restricted to piece-plotted shells from the same excavation contexts as beads or preforms, with a sample of up to four (if available) selected from each context.

In the absence of sediment samples for each aggregate, the unmodified LSS without adhering sediment provided control for what proportion of Fe to expect in the shell alone. The pXRF measurements are shown as weight $\%$ (Wt\%), describing the frequency of iron measured in Parts Per Million (ppm). Scan data were then subjected to Kruskal-Wallis tests for equal medians and Dunn's post hoc tests using PAST software (Hammer et al. 2001). Specimens coded by JM as probable preforms were included in the pXRF analysis as preforms. We also scanned 160 specimens of archeological pigments recovered from the same sites, to serve as a guide for the iron content that would be expected in red-colored pigments from these contexts.

To further examine the source of iron readings from the pXRF, we selected a subsample of 22 archeological specimens for analysis with a scanning electron microscope (SEM). The sample comprised 7 with no visible red residues nor visible matrix, 8 with visible red residues but no matrix, 


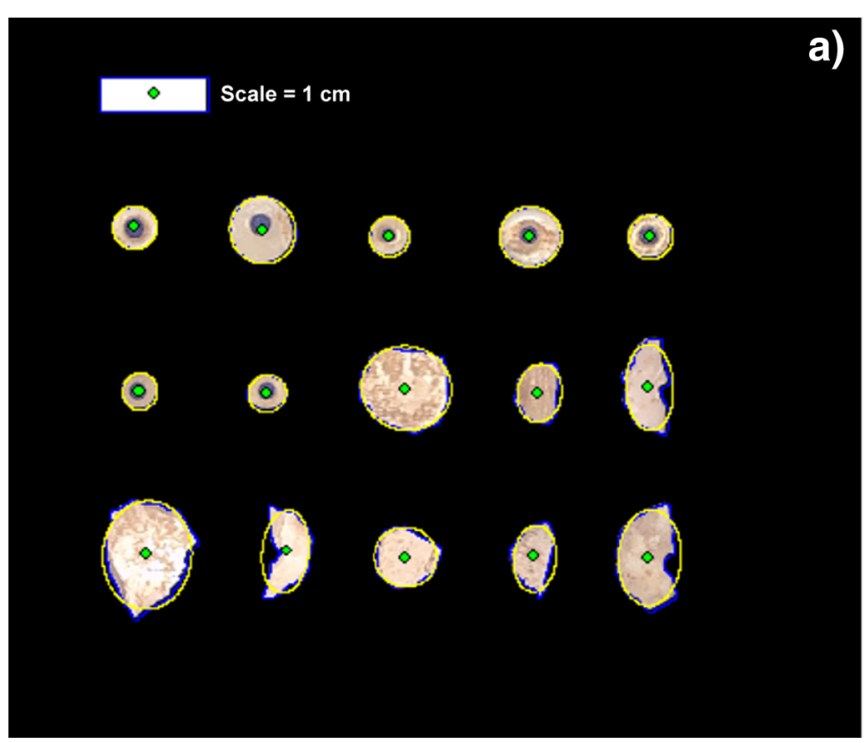

Fig. 3 Screenshot from Tomato Analyzer showing beads and preforms from Mazinga 1 (a) and close-up of a preform (b). Each artifact is outlined in blue, and the surface area reported is the area bounded by that outline. The green dots mark the center of each artifact. The yellow ovals are the

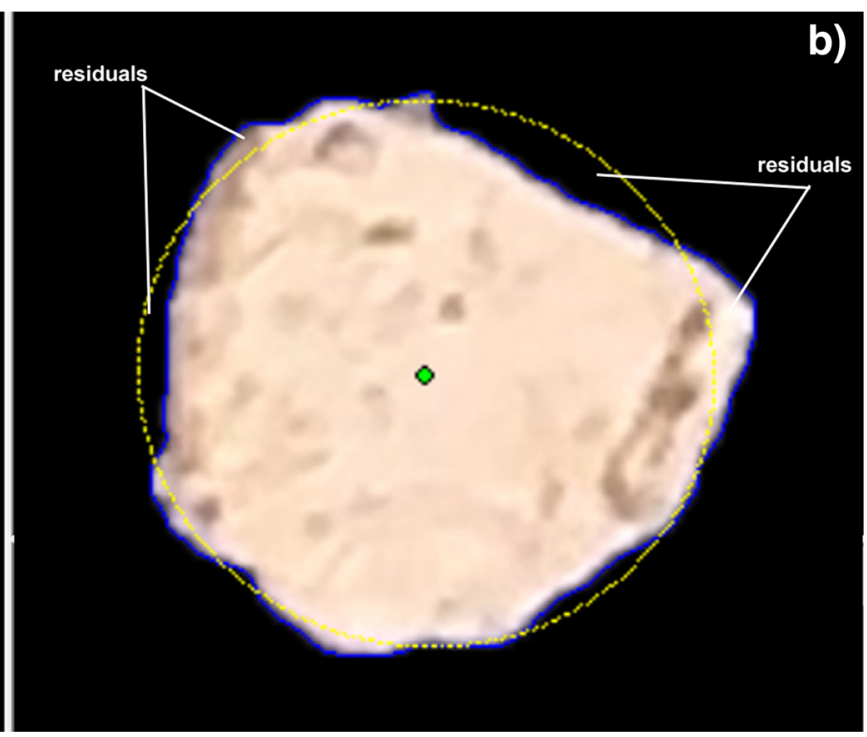

ellipses of best fit used to calculate elliptical homogeneity, which is a function of the residuals (marked in b) that depart from the ellipse of best fit. An elliptical/circular homogeneity score of 0 would reflect a perfect fit and 7 with visible matrix but no red residues. We targeted scans of the visible residues, adhering matrix, and clean surfaces of the shell as applicable (not every specimen offered the same opportunities). We used a Thermo Phenom XL G2 SEM to analyze the Fe content of the clean LSS surface, potential pigment patches, and adhering matrix. Examination occurred using its backscattered electron detector under a low vacuum and at an acceleration voltage of $15 \mathrm{kV}$. Targeted spots (e.g., exposed surface, pigment, and matrix) on each specimen were analyzed at least twice using the integrated energy-dispersive spectrometry (EDS) system, and quick elemental maps (5 min acquisition time) were subsequently collected of the surrounding areas. This map (typically 400-600 $\mu \mathrm{m}$ ) encompassed the targeted areas and provided a more general picture of the distribution of elements across the features of the surface. Because the map readings generate average elemental values across the surface, this offers a more direct comparison to the pXRF data in comparison to a spot scan in the SEM (Supplementary Fig. 2).

\section{Dating}

We sent two specimens, one each from the HOR-1 and MAZ-1 sites, to the Center for Applied Isotope Studies in Athens, Georgia, for direct AMS ${ }^{14} \mathrm{C}$ dating (Fig. 4). Preforms were selected in both cases to minimize the destruction of finished beads, which can contain substantial cultural information. In both cases, we submitted the stratigraphically lowest examples of confirmed LSS manufacturing debris recovered during the 2017 field season. Since that time, we have recovered specimens in deposits up to $45 \mathrm{~cm}$ and $60 \mathrm{~cm}$ lower at HOR-1 and MAZ-1, respectively. Although associated ${ }^{14} \mathrm{C}$ ages on unmodified land snail shell and charcoal from the same deposits are all Holocene, our results should be considered minimum ages for the antiquity of the tradition of LSS disc bead manufacture.

During dating pretreatment, the shell samples were acid-etched with dilute $\mathrm{HCl}$ to remove the outer shell layer to minimize the effects of secondary or diagenetic carbonates, rinsed in ultrapure water, and dried at $60^{\circ} \mathrm{C}$. The pretreated samples were crushed and reacted with $100 \%$ phosphoric acid in evacuated reaction vessels to produce $\mathrm{CO}_{2}$, which was cryogenically purified from the other reaction products. $\mathrm{CO}_{2}$ samples were catalytically converted to graphite using the method of Vogel et al. (1984). Graphite ${ }^{14} \mathrm{C} /{ }^{13} \mathrm{C}$ ratios were measured using the $0.5 \mathrm{MeV}$ accelerator mass spectrometer. The sample ratios were compared to the ratio measured from the Oxalic Acid I standard (NBS SRM 4990). Carrara marble (IAEA C1) was used as the background, and travertine (IAEA C2) was used as a secondary standard. The results are presented as percent Modern Carbon (pMC), and the quoted uncalibrated dates are given in radiocarbon years before 1950 (years BP), using the ${ }^{14} \mathrm{C}$ half-life of 5568 years. The error is quoted as one standard deviation and reflects both statistical and experimental errors. The dates have been corrected for isotope fractionation based 
Fig. 4 Directly dated specimens with unique specimen identifiers 35699 from HOR-1 and 13776 from MAZ-1
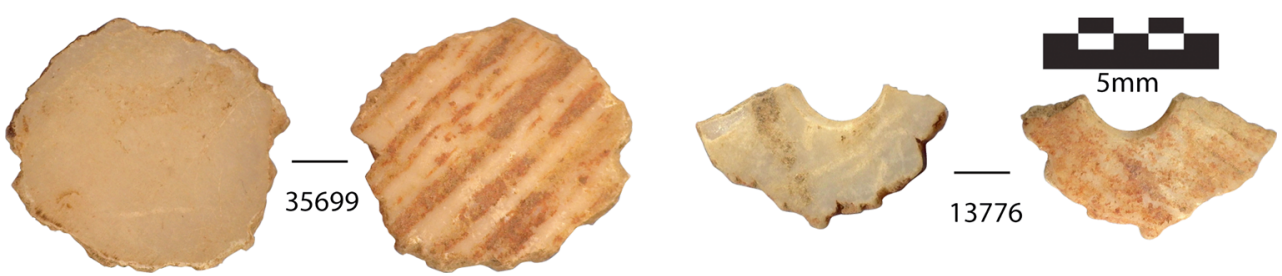

on the $\delta^{13} \mathrm{C}$ values measured by IRMS. Calibration was done using the SHCal20 curve in OxCal 4.4 (Hogg et al. 2020).

\section{Results}

\section{Experimental results}

\section{Breakage}

The modern shells from Florida used in this experiment had a maximum thickness of $1.47 \mathrm{~mm}$ at the apertural lip, which is thinner than some of the archeological beads that reach as much as $1.60 \mathrm{~mm}$. This difference in thickness between the archeological and modern shells is expected, as the Florida specimens are mostly juveniles. Despite the thinness of the modern shells, they are still remarkably resistant to breakage. To achieve breakage under body weight, each shell had to be crushed individually, requiring an excess of $90 \mathrm{~kg}(200 \mathrm{lbs})$ of directed force. Even with this technique, one shell could not be broken and remained intact. Not only is the shell tough, but it is also sharp, and jagged pieces tore easily through the cloth and paper bags that were used to keep the fragments together. The trampling simulation produced a range of shell fragments from $\sim 5 \mathrm{~cm}$ to powderized dust. Large pieces of the body whorl were still identifiable, and all apices remained intact. Shapes of the naturally broken shell tended to be linear or roughly rectangular and also included a variety of square, triangular, and partially rounded fragments. A few pieces had a smooth, rounded edge that simulated the appearance of shaping, but none were rounded on more than $50 \%$ of the entire circumference. Some parts of the shell layers came apart, and there were several dozen small $(<1 \mathrm{~mm})$ semitransparent or iridescent pieces which may have derived from a single specimen.

Using the ball-peen hammer, or hammer with a metal chisel, we were unable to break the body whorl into suitable fragments. The directed force was able to break through the shell, but only in the impact area, resulting in fragments that were too small for use as preforms. Some of the shell fragments from these processes did resemble early-stage preforms, but most were too small in diameter to replicate the bead production observed in the archeological samples. The most effective technique for intentionally breaking the shells was using a stone hammer and anvil. Similar to the trampling breakage, this produced fragments that were rectangular, square, triangular, or even semirounded.

\section{Shaping}

Rough shaping of the LSS could be achieved through snapping off protruding edges by hand, but this tended to break the specimens across the center. The finer trimming to create disc shapes could not be accomplished without tools. Hard hammer percussion while holding the LSS in the free hand was unsuccessful at removing any material; blows from the ball-peen hammer and rock both just glanced off the edge of the shell. Indirect percussion using an anvil, a metal chisel to direct force, and a hammer/rock was able to break the shell but broke off pieces that were too large, instead of neatly trimming the edge into a disc. Rubbing the edges of the shell with sandpaper or granite both created a smooth, regular edge, but these grinding techniques were likely used in final shaping stages and were not responsible for the appearance of the scalloped and trimmed edges observed in the archeological preforms.

The only method that simulated the appearance of archeological LSS preforms prior to grinding was pressure flaking. Pressure flaking the edges of an LSS preform requires strength but little skill and can accurately trim away the unwanted material. It was slightly easier to trim with the inner surface of the preform facing upward, as when the outer surface was facing upward, the slight convexity of the shell tended to make the tool slip against the domed surface. When holding the pressure flaker perpendicular to the preform, the resulting removals created an indentation approximately the width of the tool. If the tool was held at a more obtuse angle, it broke off undesirably large pieces or split the piece, which sometimes rendered the preform useless.

Our experiments revealed that semirounded fragments can be unintentional byproducts of shell breakage, although no fully rounded pieces were created by accident. This strongly suggests that any shell with a fully rounded shape has been deliberately fashioned, especially if it has scalloped edges showing damage associated with pressure flaking. Figure 5 shows experimental preforms compared to archeological preforms. 


\section{Drilling}

Experimental drilling often excludes analysis of the force applied to a shell (Tátá et al. 2014; Wei et al. 2017; Werner and Miller 2018), but Nigra and Arnold (2013) have demonstrated that $1360 \mathrm{~g}$ (3 lbs) of pressure is sufficient to drill marine shell beads. We used this value as a baseline and found that all LSS fragments $<0.50 \mathrm{~mm}(N=12)$ broke before reaching $1360 \mathrm{~g}$ of pressure. Those fragments thicker than $0.50 \mathrm{~mm}$ were capable of withstanding $1360 \mathrm{~g}$ of force. Six of the 18 fragments $\geq 0.50 \mathrm{~mm}$ broke between 1400 and $1800 \mathrm{~g}$, while the remaining 9 exceeded $1900 \mathrm{~g}$ of force and did not break. This indicates that LSS thinner than $0.50 \mathrm{~mm}$ were not likely to survive perforation. Numbers under each specimen are unique specimen identifiers.

\section{Production stages specific to LSS}

The manufacturing stages for OES beads defined by Orton (2008) translate well to LSS, but slight adjustments should be made to account for the differences between the shell structures and functions (Table 2).

Our experimental results indicate that extreme caution must be used when identifying early-stage PW2 LSS preforms. At sites where LSS also occurs as potential food debris or natural accumulations, Stage I preforms (a simple broken fragment of shell) cannot be reliably identified as anthropogenic. Although intentionally shaped pieces tend more towards round shapes with obtuse angles, natural breakage can also create similar shapes. Therefore, pieces of shell that are thin will be more prone to breakage around the edges and may trend towards
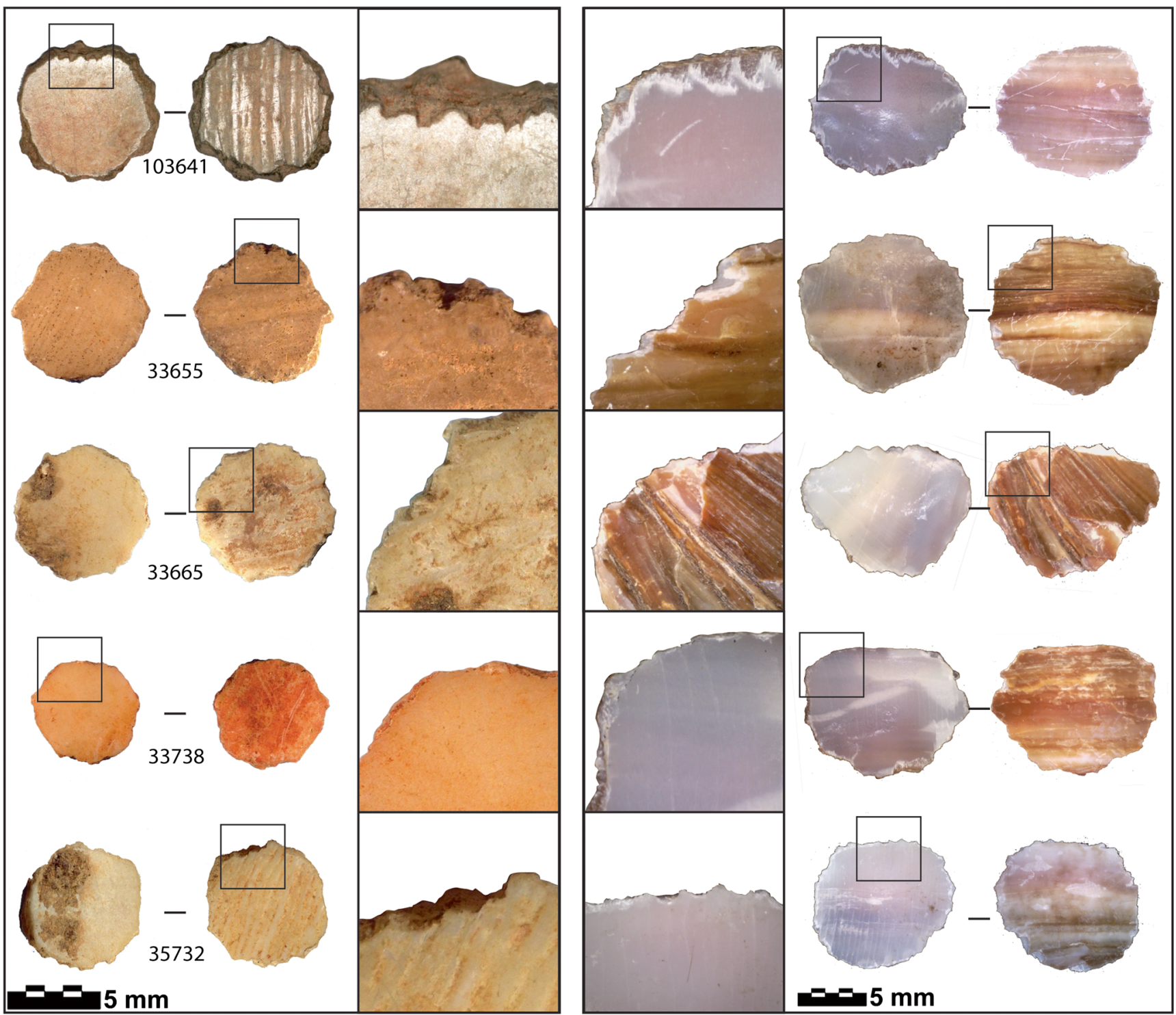

Fig. 5 Archeological PW2 Stage III preforms from HOR-1 (left) compared to experimental preforms at the same stage (right). Boxes show close-ups of edges with similar scalloping from pressure flaking 
round shapes as acute angles are naturally broken off, while thicker shells will withstand greater force.

With these caveats in mind, we suggest two main considerations be applied when identifying PW2 Stage II LSS preforms: shell thickness (most critical) and percentage of shaping. Although LSS fragments thinner than $0.50 \mathrm{~mm}$ may have been intended as preforms, we do not recommend that they be treated as preforms even if they appear to be shaped. Shells thinner than this are not capable of standing up to the forces of drilling; they may have been shaped into a disc for another purpose, or potentially created by people who were learning or practicing (Arnold 2012). This threshold for thickness established from experimental data is supported by the archeological bead data; the thinnest completed LSS bead is $0.56 \mathrm{~mm}$. However, we still encourage caution even for thicker specimens. The second consideration is that a PW2 LSS Stage II preform should be at least 50\% shaped to help rule out some degree of natural taphonomic rounding. In our analysis, all specimens assigned to "probable preform" status were PW2 Stage II or III specimens that met both the thickness criterion and the $>50 \%$ shaping criterion.

Although other stages remain largely the same as for OES, deciding when a bead has reached the end of production is a subjective decision, both on the part of the beadmaker and the researcher. Future experimental work would help conclusively determine the effects of use wear on LSS beads, but the presence of a glossy patina should not be part of the definition of a completed LSS bead. Unlike OES, the cross-layered structure of LSS may cause them to wear in a way that exposes the layers and creates a rough or even "ground" appearance, instead of producing a high shine.

\section{Application of pigments}

Modern specimens of giant land snail shells have very low quantities of iron, as detected by pXRF. After application of ochre, the pXRF detected significantly more iron in modern specimens, and this level remained high (although significantly less), once the specimens were washed $(p<0.0001)$ (Fig. 6). This simulates the removal of ochre through processes such as sediment leaching or even screen-washing during recovery. The red archeological pigments have a very large range of variation in Fe content (Wt\% between 3 and 64), which overlaps at its lower end with the experimentally ochred shells but not with shells that had been washed.

\section{Archeological results}

\section{Assemblage description}

A summary of the production stages and pathways for each site is provided in Table 3. It is notable that LSS beads and preforms are not found in confirmed Pleistocene deposits at any of the sites.

Based on our experimental work, production Stage I specimens (a simple broken fragment of shell with no shaping or drilling) cannot be differentiated from naturally fragmented shells. All production stages after this are represented at HOR-1, while MAZ-1 and KAD-1 are missing some of the intermediate production stages where drilling or grinding have begun but are incomplete (Stage VI at MAZ-1 and Stages IV and VI at KAD-1). This is likely because of their smaller sample sizes, which may in turn be a function of excavated sediment volume of the right geochronological age. At HOR1 , about half the excavated sediment volume is Early Holocene, the majority of excavated sediment at MAZ-1 is Pleistocene, and all the excavated sediment at KAD-1 is Late Holocene. The presence of early manufacturing stages at all three sites indicates that LSS beads were manufactured at each, even if the clearest signatures of this behavior are at HOR-1.

\section{Manufacturing chains}

Intuitive parallels between OES and LSS bead production appear to bear out in the Malawi assemblages, and LSS bead production follows the same general sequences as OES bead manufacture. Similar to the chaîne opératoire used for OES beads, LSS beads likewise can follow either PW1 or PW2. This is perhaps unsurprising as there are a limited number of ways to produce a disc bead from a flat piece of shell, but these artifacts provide the first confirmation of these constraints in two different raw material types (Fig. 7).

Based on experimental replication, the primary means of shaping appears to be pressure flaking the edges of an angular Stage I preform into a rounded shape. Archeological and ethnographic evidence for OES beadmaking is overwhelmingly represented by $\mathrm{PW} 1$ production, in which the shaping is done after the drilling. While both are occasionally used, many sites will exclusively have PW1 OES preforms (Orton 2008). Surprisingly, LSS preforms at the Malawi sites are dominated by PW2 manufacture, in which shaping takes place prior to drilling. Of the specimens confidently identified as preforms that could be assigned to a pathway (i.e., had not progressed to a manufacturing stage that erased evidence of the original pathway), $90.1 \%$ at HOR-1 $(N=101), 100 \%$ at MAZ-1 $(N=16)$, and $100 \%$ KAD-1 $(N=2)$ were PW2.

Drilling a perforation was the next step in a PW2 production sequence. As long as the inner (iridescent) or outer (ridged) shell layer is present and the aperture has not been excessively enlarged by wear, it can be possible to determine from which surface drilling it originated (see Werner and Miller (2018) for discussion of drilling). For beads where the direction of drilling could be established, there was a clear preference for either drilling from the inside or both sides, rather than from the outside only. At 


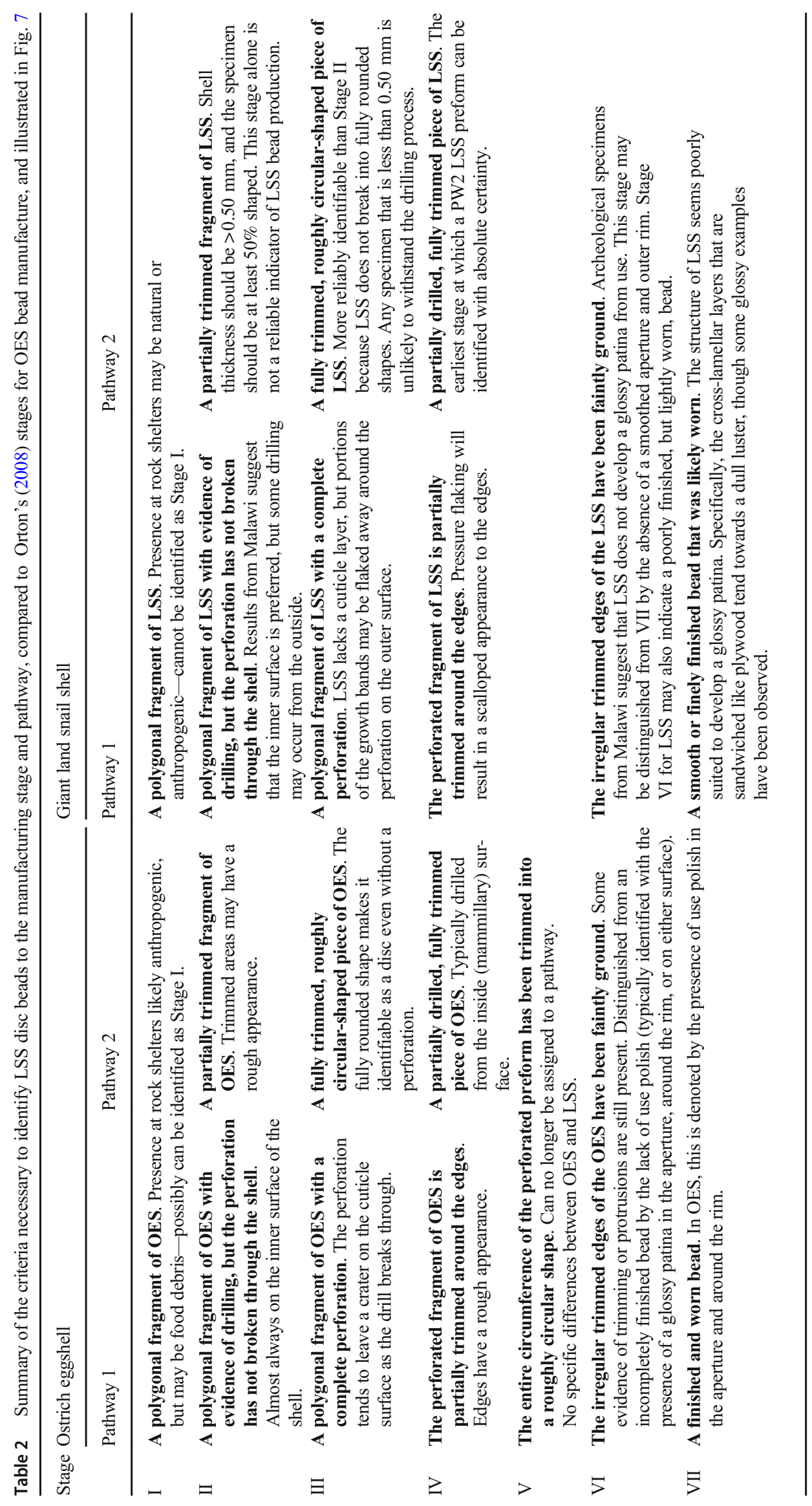




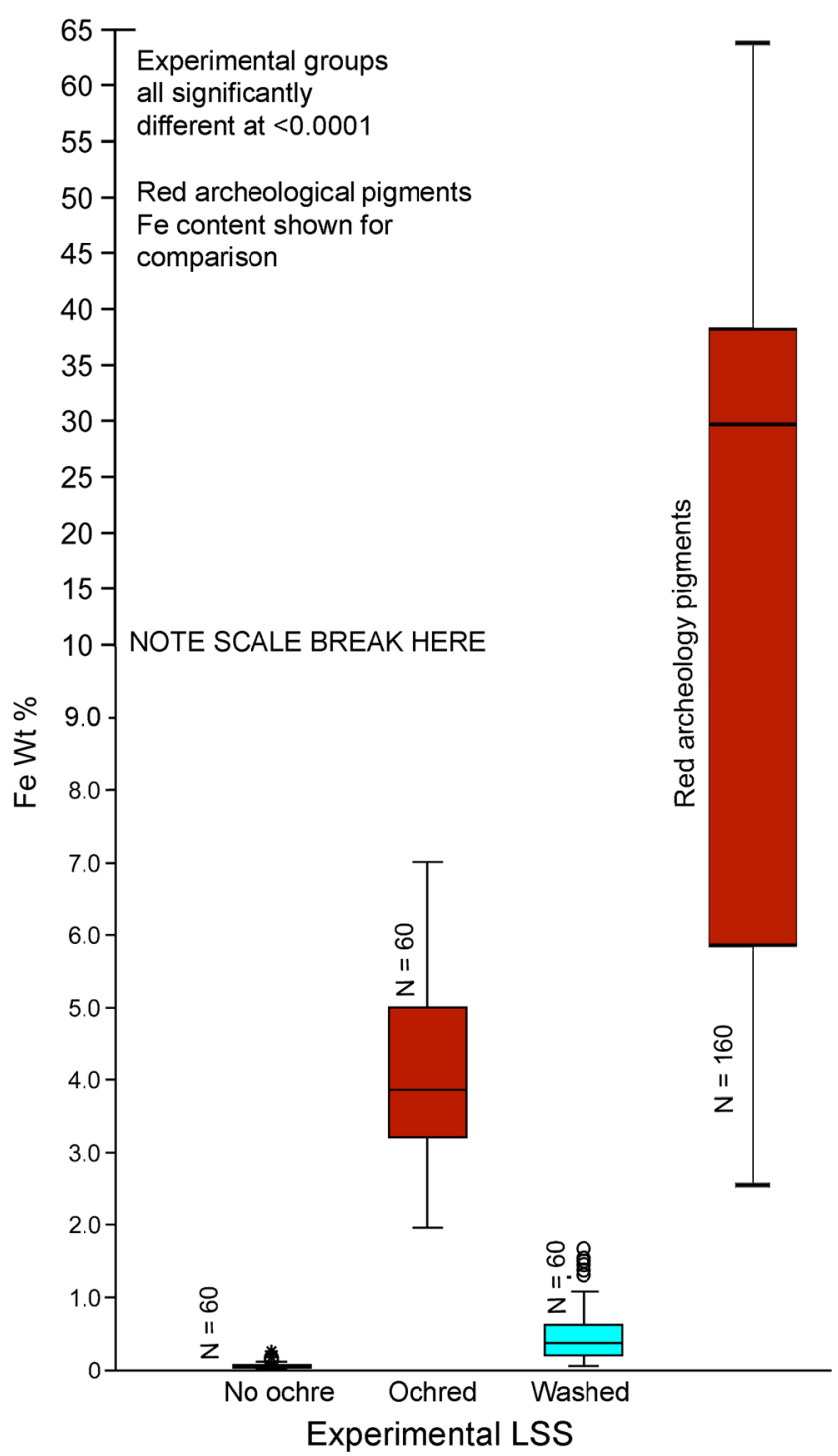

Fig. 6 Box plots showing pXRF measurements of Fe Wt\% for modern giant land snail shells without ochre, after application of ochre, and after washing, in comparison to the $\mathrm{Fe} \mathrm{Wt} \%$ of red archeological pigments from the same sites

HOR-1 $(N=86), 69.8 \%$ were drilled from the inside, $5.8 \%$ from the outside, and $24.4 \%$ from both. At MAZ-1 $(N=22), 45.5 \%$ were drilled from the inside and $54.5 \%$ were drilled from both, and at KAD-1 $(N=4), 75 \%$ were drilled from the inside and $25 \%$ from both.

Substantial material was removed from the shaped and drilled preforms before finishing the final product. Morphological attributes by site and production stage for HOR-1 and MAZ- are reported in Table 4, with results of the Kolmogorov-Smirnov tests in Supplementary Table 1. Only complete, unbroken LSS preforms and beads are included (Fig. 3), and all measurements provided in Table 3 are in $\mathrm{cm}$. The surface area is reported in $\mathrm{cm}^{2}$, defined as the area within the outline of the preform, including the hole in perforated specimens. Maximum width and maximum height are
Table 3 Production stages and pathways for LSS disc beads from the three sites, using the modified Orton (2008) sequence including $a / b$ for complete/broken

\begin{tabular}{lllll}
\hline & Site & & & \\
\cline { 2 - 4 } Stage & HOR- & KAD- & MAZ- & Total \\
& 1 & 1 & 1 & \\
\hline IIa & 17 & 0 & 5 & 22 \\
IIb & 8 & 0 & 1 & 9 \\
IIIa & 40 & 2 & 2 & 44 \\
IIIb & 19 & 0 & 4 & 23 \\
IVa & 4 & 0 & 0 & 4 \\
IVb & 14 & 0 & 3 & 17 \\
Va & 5 & 0 & 0 & 5 \\
Vb & 8 & 1 & 4 & 13 \\
VIa & 4 & 0 & 0 & 4 \\
VIb & 1 & 0 & 0 & 1 \\
VIIa & 46 & 8 & 19 & 73 \\
VIIb & 22 & 4 & 3 & 29 \\
Total & 188 & 15 & 41 & 244 \\
\hline
\end{tabular}

reported as measured by a bounding rectangle. The shape index is the ratio of maximum height to maximum width. Ellipsoid homogeneity and circular homogeneity quantify roundedness of the preforms in terms of residuals based on an ellipse or circle of best fit, respectively (Heckel 2016).

The data show that at HOR-1, on average, $41 \%$ of area was lost between Stage II and Stage V (the point at which pathways become indeterminate), and $60 \%$ was lost between Stage II and the finished Stage VII. At MAZ-1, 73\% of the average area was removed between Stage II and Stage VII. Although these basic differences in descriptive statistics are apparent, in most cases small sample sizes preclude meaningful statistical comparisons between sites and stages (the sample from KAD-1 is too small for even descriptive statistics to be useful). The exception is Stages II and III (PW2), and Stage VII (PW indeterminate) from HOR-1, where the samples of II $(N=16)$, III $(N=40)$, and VII $(N=9)$ specimens allow for comparison in independent-sample $t$-tests (Table 5). Because the sample size of Stage III is more than twice as large as that of Stage II, effect size was calculated as Hedges' $g$. In all cases, effect sizes exceed the threshold for a strong effect, which is 0.8 . Statistically significant differences exist between Stage II preforms and Stage III preforms for all variables except the shape index. Stage III preforms are smaller than Stage II preforms in terms of width, height, and area, and more rounded in terms of both elliptical and circular homogeneity. Width and height are also significantly smaller as Stage III preforms progress into Stage VII.

The assumption of homogeneity of variance was not met for Stage III and Stage VII in the variables of ellipsoid homogeneity, circular homogeneity, and shape index 
Fig. 7 Production stages for LSS beads showing Pathway 1 and Pathway 2 variations, with archeological examples shown from Stages II-VII. Pathway 2 Stages II and III are from MAZ-1; all others are from HOR-1. Images not to scale. Numbers under each specimen are unique specimen identifiers
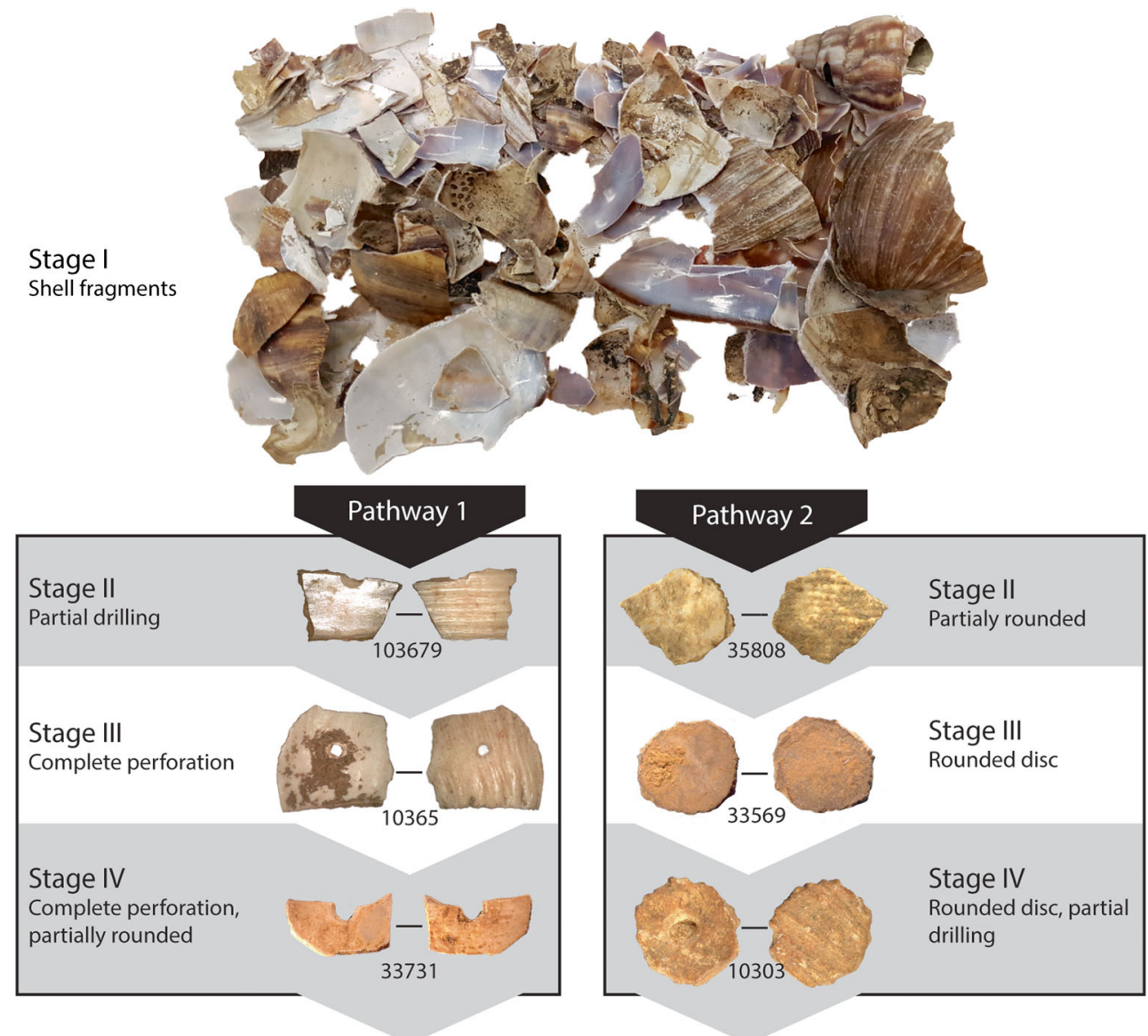

Pathway indistinguishable

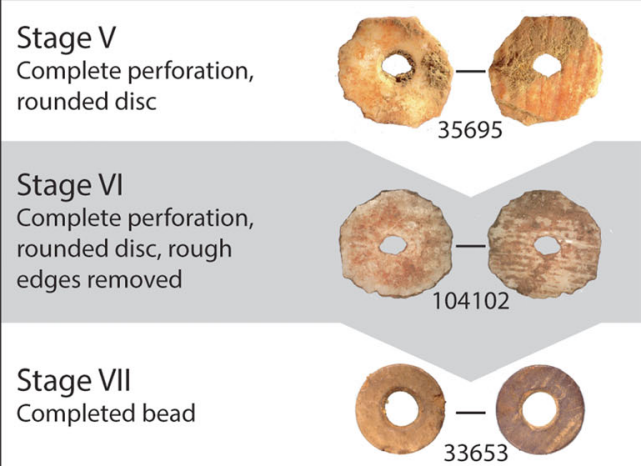

(Supplementary Table 2). However, box plots in Fig. 8 show that finished beads (Stage VII) are more rounded and refined in outline than are Stage III preforms, in terms of both ellipsoid homogeneity and circular homogeneity. For the shape index, the range displayed by finished beads falls within that of Stage III preforms but is more restricted. Overall, morphometric analyses completed by $\mathrm{CH}$ show that the typological classifications provided by JM are not only valid but show statistically significant between-group differences with a reduction in size and greater refinement of the ellipsoid/circular outline from Stage II to Stage III to the finished bead (Stage
VII). Future analyses of archeological and experimental samples will focus on developing quantitative standards for preform typology.

Red residues seem to have been introduced onto the surfaces of specimens during all stages of production. These residues took the form of thin or thick discontinuous layers, clumps, or specks on the inside or outside or in the aperture of the specimens. At HOR-1, 42 beads, 47 preforms, and 3 probable preforms (48.6\% of all specimens), at MAZ-1, 2 beads, 5 preforms, and 1 probable preform (19.5\%) of specimens, and at KAD-1 $3(20.0 \%)$ of specimens had some 
Table 4 Descriptive statistics for the morphological attributes of bead preforms and completed beads from the HOR-1 and MAZ-1 LSS assemblages. Only unbroken specimens are included

\begin{tabular}{|c|c|c|c|c|c|c|c|c|c|c|c|}
\hline & \multirow[b]{3}{*}{ Stage } & \multicolumn{7}{|l|}{ Hora 1} & \multicolumn{3}{|c|}{ Mazinga 1} \\
\hline & & \multirow{2}{*}{$\begin{array}{l}\text { PW1 } \\
\text { IV } \\
(n=1)\end{array}$} & \multicolumn{3}{|l|}{ PW2 } & \multirow{2}{*}{$\begin{array}{l}\text { PW indeterminate } \\
\mathrm{V} \\
(n=5)\end{array}$} & \multicolumn{2}{|c|}{ Complete beads } & \multicolumn{2}{|l|}{ PW2 } & \multirow{2}{*}{$\begin{array}{l}\text { Complete beads } \\
\text { VII } \\
(n=20)\end{array}$} \\
\hline & & & $\begin{array}{l}\text { II } \\
(n=16)\end{array}$ & $\begin{array}{l}\text { III } \\
(n=40)\end{array}$ & $\begin{array}{l}\text { IV } \\
(n=3)\end{array}$ & & $\begin{array}{l}\mathrm{VI} \\
(n=3)\end{array}$ & $\begin{array}{l}\text { VII } \\
(n=27)\end{array}$ & $\begin{array}{l}\text { II } \\
(n=5)\end{array}$ & $\begin{array}{l}\text { III } \\
(n=2)\end{array}$ & \\
\hline \multirow[t]{2}{*}{ Area $\left(\mathrm{cm}^{2}\right)$} & Mean & 0.352 & 0.572 & 0.397 & 0.418 & 0.340 & 0.486 & 0.228 & 0.599 & 0.392 & 0.160 \\
\hline & (st dev.) & $(\mathrm{n} / \mathrm{a})$ & -0.180 & -0.133 & -0.077 & -0.136 & -0.173 & -0.085 & -0.318 & -0.050 & -0.112 \\
\hline \multirow[t]{2}{*}{ Maximum width $(\mathrm{cm})$} & Mean & 0.659 & 0.841 & 0.718 & 0.741 & 0.732 & 0.788 & 0.567 & 0.893 & 0.757 & 0.435 \\
\hline & (st dev.) & $(\mathrm{n} / \mathrm{a})$ & -0.170 & -0.124 & -0.075 & -0.118 & -0.127 & -0.097 & -0.241 & -0.075 & -0.134 \\
\hline \multirow[t]{2}{*}{ Maximum height (cm) } & Mean & 1.007 & 0.863 & 0.718 & 0.758 & 0.778 & 0.822 & 0.567 & 0.893 & 0.690 & 0.432 \\
\hline & (st dev.) & $(\mathrm{n} / \mathrm{a})$ & -0.183 & -0.121 & -0.069 & -0.133 & -0.137 & -0.095 & -0.220 & -0.041 & -0.134 \\
\hline \multirow[t]{2}{*}{ Shape index (ratio $h: w$ ) } & Mean & 1.529 & 1.028 & 1.003 & 0.992 & 1.063 & 1.042 & 0.999 & 1.012 & 0.914 & 0.992 \\
\hline & (st dev.) & $(\mathrm{n} / \mathrm{a})$ & -0.080 & -0.084 & -0.033 & -0.427 & -0.007 & -0.022 & -0.123 & -0.036 & -0.035 \\
\hline \multirow[t]{2}{*}{ Ellipsoid homogeneity } & Mean & 0.116 & 0.050 & 0.034 & 0.034 & 0.070 & 0.029 & 0.009 & 0.053 & 0.039 & 0.010 \\
\hline & (st dev.) & $(\mathrm{n} / \mathrm{a})$ & -0.009 & -0.011 & -0.007 & -0.037 & -0.009 & -0.004 & -0.006 & -0.016 & -0.003 \\
\hline \multirow[t]{2}{*}{ Circular homogeneity } & Mean & 0.287 & 0.064 & 0.042 & 0.039 & 0.072 & 0.031 & 0.010 & 0.068 & 0.047 & 0.014 \\
\hline & (st dev.) & $(\mathrm{n} / \mathrm{a})$ & -0.014 & -0.018 & -0.007 & -0.058 & -0.006 & -0.004 & -0.009 & -0.016 & -0.010 \\
\hline
\end{tabular}

amount of red residue. However, specimens from HOR-1 had more "substantial" pigment coverage with 55/188 specimens (29.2\%), compared to MAZ-1 with only $2 / 41$ specimens (4.9\%), and at KAD-1 with only $1 / 15$ specimens (6.7\%).

Figure 9a shows that the visual attribute of "substantial" red residues corresponds to significantly more iron content than specimens without this attribute $(H=69.65$; $p<0.0001)$. Specimens with sediment on $\geq 30 \%$ of the surface were excluded from this analysis, to determine if the pXRF can detect an increase in Fe when there is residue present, without potential interference from $\mathrm{Fe}$ in the sedimentary

Table 5 Results of one-tailed independent sample $t$-tests from HOR-1 comparing PW2 Stage II and Stage III preforms (a) and comparing Stage II/III preforms with Stage VII finished beads (b). DF refers to degrees of freedom

(a)

\begin{tabular}{lccll}
\hline Attribute & DF & $t$-value & $p$ value & Hedge's $g$ (effect size) \\
\hline Area & 55 & 4.01 & 0.000092 & 1.19 \\
Ellipsoid & 55 & 5.39 & $<0.00001$ & 1.34 \\
Circular & 55 & 4.21 & 0.000048 & 1.25 \\
Max width & 55 & 3.55 & 0.000401 & 1.05 \\
Max height & 55 & 4.19 & 0.000052 & 1.24 \\
Index & 55 & 1.24 & 0.10961 & $\mathrm{n} / \mathrm{a}$
\end{tabular}

(b)

\begin{tabular}{lllll} 
Attribute & DF & $t$-value & $p$ value & Hedge's $g$ (effect size) \\
Area & 64 & 5.76443 & $<0.00001$ & 1.45 \\
Max width & 64 & 5.24161 & $<0.00001$ & 1.322 \\
Max height & 64 & -5.36342 & $<0.00001$ & 1.35 \\
\hline
\end{tabular}

matrix. Dunn's post hoc tests show that the iron content of LSS without any visible red residues is significantly different from LSS with both trace $(p=0.0092)$ and substantial $(p<0.0001)$ amounts of residues. Further, specimens with substantial residues have a $\mathrm{Wt} \%$ of Fe significantly different from specimens with only trace residues $(p<0.0001)$. This confirms that red staining can be used to visually identify iron-containing residues and that the pXRF can measure the approximate proportions of iron on the surfaces of LSS. It is notable that as shown in Fig. 9b, the pXRF detected a very similar distribution of Fe values between archeological specimens with substantial red residues and experimental specimens that had ochre applied and were later washed. Most archeological specimens were recovered during wet-sieving and yet still retained these visible residues.

Next, we considered the possibility that iron was introduced to the surfaces of LSS via the surrounding sediment, which has become incorporated onto some specimen surfaces by an adhering matrix. We compared specimens with substantial red residues and little matrix coverage to one another and then compared specimens with little/no red residue but substantial matrix coverage to one another $(\geq 30 \%$ of their surfaces). Figure $9 \mathrm{~b}$ shows that although the larger sample size of scans of unmodified LSS with substantial sediment resulted in a number of outliers, the median $\mathrm{Wt} \%$ of iron in the unmodified LSS is lower than that in any of the other samplesregardless of matrix coverage $(H=41.8 ; p<0.0001)$. Dunn's post hoc tests show that the Fe content of unmodified LSS is significantly lower than that of both preforms and beads but that there is no significant difference between preforms and beads (Table 6). 
Fig. 8 Box plots of ellipsoid homogeneity, circular homogeneity, and shape index, which did not meet the assumption of homogeneity of variance for Stage VII

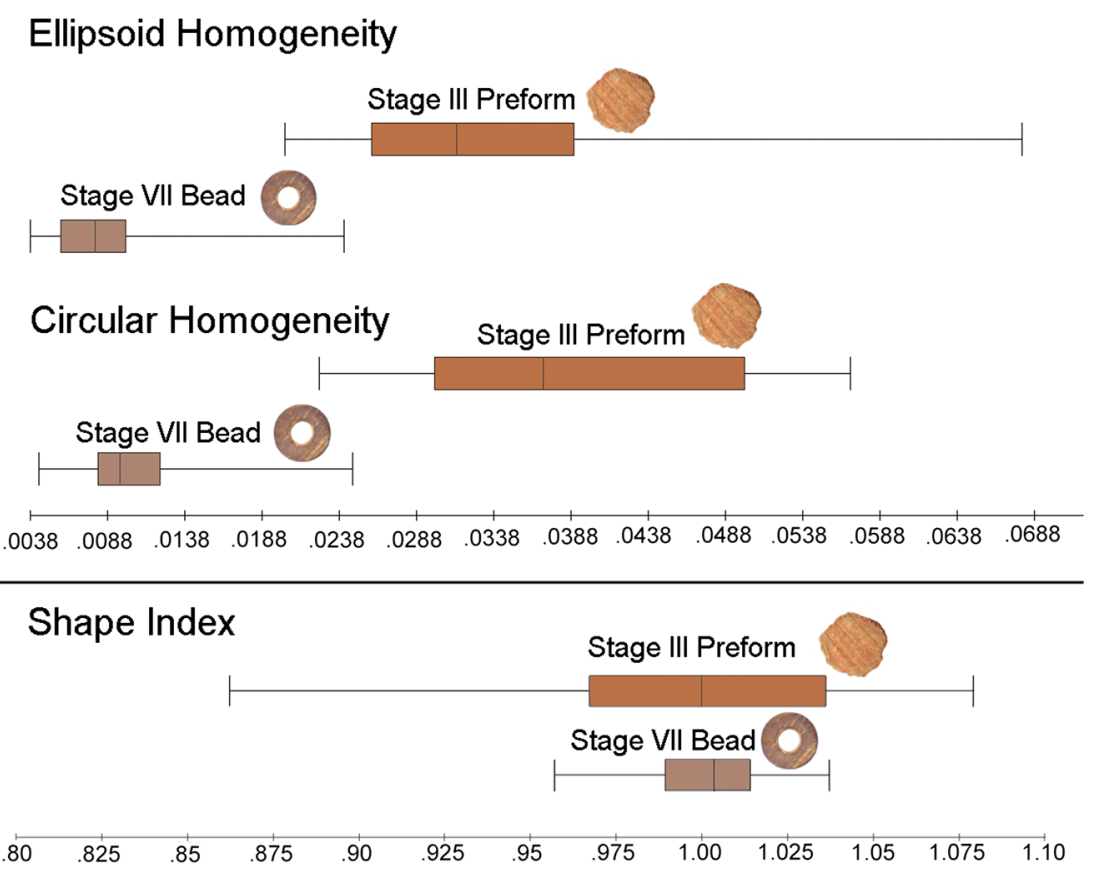

Because matrix coverage may have an effect on $\mathrm{Wt} \%$ of Fe, Fig. 9c shows specimens with $\geq 30 \%$ matrix coverage only, broken into groups with substantial red residues versus none/ trace residues. In spite of all having substantial matrix coverage, specimens with substantial residues still have a significantly higher median $\mathrm{Wt} \% \mathrm{Fe}$ than specimens with none/trace residues (Mann-Whitney $z$-score: $3.5945 ; p=0.0003$ ).

SEM data (Fig. 9d) confirm our inferences from pXRF that the adhering sediment/matrix can contribute to elevated Fe readings, but that there is a significant difference between the iron content in residues and sediment $(H=26.55, p<0.0001)$. While there is some overlap, the higher frequency of iron present in the red residues approaches the content in red archeological pigments recovered from the site. This suggests that the application of ochre will be visible when measuring quantities of iron. The mapping captures variations in surface elements and averages them across the mapped area, which explains the reduction in iron content between mapped values and targeted values even on specimens with large amounts of residue (Table 7). When compared directly to the pXRF readings, mapped SEM values are overall higher. This is expected because the SEM is not averaging values across the entire specimen, and confirms that the pXRF is indeed a blunt instrument. However, its utility as a quick, affordable option for confirming elevated Fe levels is demonstrated by the strong correlation between rank-ordered Fe readings using the two approaches (Spearman's Rs = $0.6480, p=0.0011$ ).

Additional evidence that pigment was incorporated onto the surface of some specimens prior to Stage IV in the PW2 production sequence comes from preforms where one or both surfaces are strongly coated in red residue but are not present in the aperture initiation. In other specimens, the pigment may have been introduced into the aperture after manufacture or during wear, rubbing off from associated clothing, skin, or fiber (Collins et al. 2020). In many cases, the outer ridges of the shell appear to have preferentially retained red residues that have otherwise worn away (Supplementary Fig. 1). Red residues occurred on both sides of 50 specimens, and for those with residues on only one side, 37 had it exclusively on the outside and only 8 had it exclusively on the inside.

\section{Dating}

Results of the ${ }^{14} \mathrm{C}$ analysis are provided in Table 8. Although no beads or preforms were directly dated from KAD-1, all excavated sediment dates to the Late Holocene. This shows that in addition to the directly dated beads from Iron Age contexts in Tanzania (Miller et al. 2018), LSS bead manufacture does occur in clear Later Stone Age contexts. At least in southern-central Africa, the tradition of using this material dates at least back to the Early Holocene, continued into the Late Holocene, and is no longer in use today.

\section{Discussion}

Despite the general similarities in production sequences, there are differences in the shell structures between LSS and OES that produce some variations. These must be taken into account when creating guidelines for analysis, as they will not translate directly between the two materials. A major distinction between the two materials is that the layers have different properties, resulting from the different functions each shell serves. OES are protective barriers that encase a developing 

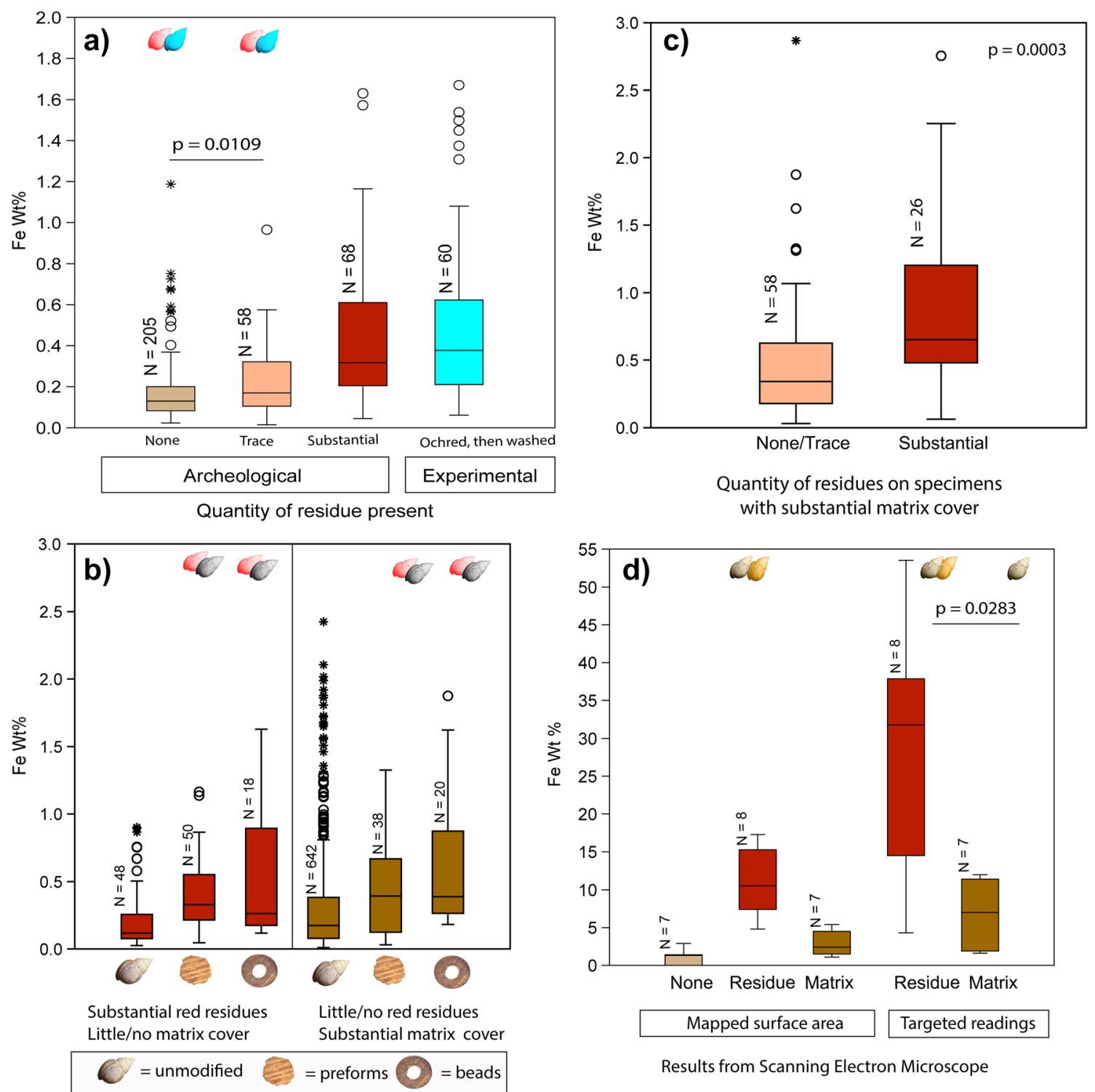

Results from Scanning Electron Microscope

Fig. $9 \mathrm{Wt} \%$ represents percent of iron ( $\mathrm{ppm})$ present in the pXRF scan. (a) Box plot showing specimens with clean surfaces, trace amounts of red residue, and substantial amounts of red residue, plus specimens from an experimental assemblage where ochre was applied and then washed with water; $p$ values between each compared pair of data are shown by connecting lines at the top of the graph. A small snail represents a significant difference (Supplementary Table 3) from the experimental assemblage (blue) or from specimens with substantial red residue (red). (b) Box plot of specimens by type (unmodified, preform, or bead) and the amount of matrix cover or residue present. Substantial matrix cover is $\geq 30 \%$ of the surface. A small snail represents a significant difference from

unmodified shell with red residue and little/no sediment (red), or from unmodified shell with sediment but little/no residue (black). Significance values for Dunn's post hoc test are in Table 6. (c) Box plot of specimens with $\geq 30 \%$ matrix cover and substantial amounts of residue compared to those with $\geq 30 \%$ matrix cover and none/trace residues. (d) Box plot of specimens scanned with the SEM, including specimens with no residues or matrix, substantial residues but no matrix, and substantial matrix but no residues. A small snail represents a significant difference from shells with no residues or matrix (gray), or from specimens with substantial matrix (gold) (Supplementary Table 4). $N$ values include two scans per specimen (one on each side)

ostrich embryo, while LSS is a living part of a gastropod. A whole ostrich egg can withstand a large amount of pressure from the outside but is able to be pecked open from inner surface when an ostrich chick is ready to hatch. This inner surface, the soft and chalky mammillary layer, contrasts with the hard but brittle cuticle layer, which is the waterimpermeable outer surface (Li-Chan and Kim 2008). On the other hand, the shell of a snail is an exoskeleton that provides a

protective covering for the slow moving and soft-bodied creature. The inner surface of a LSS is extremely smooth and sometimes iridescent, while the outer layer may be roughly textured, with parallel ridges indicating growth increments. The spiral shaping, as well as the internal cross-lamellar layers, make gastropod shells highly resistant to breakage from either internal or external forces. In sum, ostrich eggs are designed to be broken, snail shells are not. 
Table 6 Dunn's post hoc test on the samples shown in Fig. 9b (raw $p$ values, uncorrected significance). Significant results are bold. "Red" indicates "substantial red residues" as defined in the text. "Matrix" indicates "substantial matrix coverage" as defined in the text

\begin{tabular}{|c|c|c|c|c|c|}
\hline & Preform, red & Bead, red & Unmod, matrix & Preform, matrix & Bead, matrix \\
\hline Unmod, red & 0.0001 & 0.0019 & 0.2981 & 0.0023 & 0.0002 \\
\hline Preform, red & & 0.8807 & $<0.0001$ & 0.737 & 0.4238 \\
\hline Bead, red & & & 0.0034 & 0.909 & 0.4286 \\
\hline Unmod, matrix & & & & 0.0025 & 0.0002 \\
\hline Preform, matrix & & & & & 0.3353 \\
\hline
\end{tabular}

These differences in structures between ostrich and snail shells revealed the need for a reworking of the OES manufacturing sequence description. OES artifact analysis often includes descriptions of traits that cannot be universally applied to other materials. For example, descriptions of the cuticle edges on the outer surface of OES are so ingrained into the production analysis that they are encoded into the production stages. For example, Stage III is identified when the final perforation causes the cuticle layer to break away from the shell (Orton 2008: 1767), but LSS does not have a cuticle layer to break away during perforation. It also appears that use wear may manifest in different ways between OES and LSS. In Stage VII, a completed OES bead is described as

Table 7 Summary of SEM results compared to pXRF for a subset of specimens. SEM results show the elemental readings across a mapped area and elemental readings from targeted areas where there was only the having the presence of use wear, which is interpreted as a glossy patina either in the aperture, around the outer rim, or on either surface of the shell. While we have observed many OES beads with an intensely patinated, almost glassy surface, the closest observed examples of LSS beads only have a dull luster, and many beads with other signs of intense wear (such as smoothed apertures and outer rims, and obliteration of the aperture cup) still appear chalky on the surface. It is possible that the cross-lamellar inner layers of LSS are not capable of developing a glossy patina. These layers have been described as having the appearance of torn plywood (Ward and Maggs 1988: 411), which is not conducive to a shiny polished surface. Instead, the cross-lamellar layers are often revealed by

shell surface, where there was a visible red residue, and where there was matrix. ID numbers are unique specimen identifiers

\begin{tabular}{|c|c|c|c|c|c|c|c|c|}
\hline ID & Type & Residue & Sediment & $\mathrm{Fe} \mathrm{Wt} \%$ (pXRF) & $\mathrm{Fe} \mathrm{Wt \%} \mathrm{(mapped)}$ & $\mathrm{Fe} \mathrm{Wt} \%$ (surface) & Fe Wt (residue) & $\mathrm{Fe} \mathrm{Wt} \%$ (sediment) \\
\hline 33658 & Bead & None & None & 0.1 & 1.4 & 0.0 & N/A & N/A \\
\hline 33751 & Bead & None & None & 0.1 & 1.0 & 0.0 & N/A & N/A \\
\hline 35757 & Bead & None & None & 0.2 & 2.9 & 0.0 & N/A & N/A \\
\hline 33749 & Preform & None & None & 0.1 & 0.0 & 0.0 & N/A & N/A \\
\hline 35763 & Preform & None & None & 0.2 & 1.3 & 0.0 & N/A & N/A \\
\hline 35812 & Preform & None & None & 0.1 & 1.3 & 0.0 & N/A & N/A \\
\hline 13725 & Preform & None & None & 0.1 & 0.0 & 0.0 & N/A & N/A \\
\hline 33712 & Bead & Substantial & None & 0.2 & 17.3 & 0.0 & 34.5 & N/A \\
\hline 33727 & Bead & Substantial & None & 0.6 & 13.1 & 0.9 & 4.3 & N/A \\
\hline 13706 & Preform & Substantial & None & 0.5 & 8.9 & 1.7 & 29.1 & N/A \\
\hline 33641 & Preform & Substantial & None & 0.2 & 4.8 & 0.0 & 11.9 & N/A \\
\hline 33730 & Preform & Substantial & None & 0.4 & 7.3 & 6.4 & 35.8 & N/A \\
\hline 33738 & Preform & Substantial & None & 0.5 & 16.0 & 2.9 & 38.5 & N/A \\
\hline 35689 & Preform & Substantial & None & 0.5 & 7.6 & 0.9 & 22.3 & 3.7 \\
\hline 35755 & Preform & Substantial & None & 0.5 & 12.2 & N/A & 53.6 & 9.3 \\
\hline 5185 & Bead & None & Substantial & 0.5 & 2.4 & 0.0 & N/A & 11.4 \\
\hline 35726 & Preform & None & Substantial & 0.6 & 4.5 & N/A & N/A & 12.0 \\
\hline 35734 & Preform & None & Substantial & 0.1 & 1.5 & N/A & N/A & 1.9 \\
\hline 35745 & Preform & None & Substantial & 0.4 & 2.1 & 0.0 & N/A & 4.3 \\
\hline 35747 & Preform & None & Substantial & 0.7 & 3.9 & 3.9 & N/A & 11.4 \\
\hline 35813 & Preform & None & Substantial & 0.4 & 1.1 & 0.0 & N/A & 1.6 \\
\hline 35,819 & Preform & None & Substantial & 0.6 & 5.4 & N/A & N/A & 7.0 \\
\hline
\end{tabular}


Table 8 Results of direct ${ }^{14} \mathrm{C}$ ages on two LSS preforms from the Kasitu Valley

\begin{tabular}{llllllll}
\hline Type & Date (Cal BP) & $\delta^{13} \mathrm{C}(\% \circ)$ & ${ }^{14} \mathrm{C}$ age & \pm & Lab sample\# & PW & Stage \\
\hline LSS preform & $9538-9442$ & -12 & 8510 & 30 & UGAMS\#36258 & 2 & $3 \mathrm{a}$ \\
LSS preform & $9425-9127$ & -11.65 & 8310 & 30 & UGAMS\#36257 & Indet. & 5b \\
\hline
\end{tabular}

grinding or wear along the edges of the shell or in the aperture. These can sometimes even resemble grinding striations on the faces of the bead, when in fact they are just the exposure of the underlying shell structure at different levels.

These structural differences cause another problem - it can be difficult to discern the inner from outer shell surfaces of LSS if the inner nacre and outer sculptural ridges have been ground off or worn away. OES beads are almost exclusively drilled from the inner surface, not as a stylistic preference, but a structural inevitability, as the inner surface is softer and easier to drill. Trying to start drilling from the hard cuticle is more challenging and results in slippage of the drill bit along the slick outer surface. Although LSS does not have a soft, chalky inner surface that would make an intuitive surface to begin drilling, it does have a rough outer surface. In spite of this, the majority of LSS beads were drilled from the inside, perhaps facilitated by the slight curvature of the shell. The choice to begin drilling from the inner surface may also have been a strategy to reduce breakage during production, as pressure can be concentrated in the drilling location by pressing the other side directly against a substrate. The direction of drilling among LSS beads should continue to be documented in other collections and pursued in experimental projects to estimate whether it is the result of structural considerations or beadmaking tradition.

Another distinction between OES and LSS that affects their interpretation is shell thickness. Ostrich shell characteristics are affected by a number of factors including diet, temperature, and humidity (Ecker et al. 2015). Even though there will be variation, even between eggs from the same nest, a single ostrich egg has a relatively consistent thickness. Experimental work by Wilmsen (2015: 93) found that a single egg ranged from 1.74 to $2.14 \mathrm{~mm}$ thick, depending on whether fragments came from the thicker aerus (rounded end), taglion (pointed end), or the thinner waist area. This suggests a variation in thickness up to $20 \%$ within a single egg. This seems substantial but is eclipsed by the variations in thickness within a single land snail shell. The thinnest part of a gastropod shell is typically at the apex of the spire, which can be paper-thin. Not only are the upper portions of a gastropod spire very thin, they are also markedly curved as the shell's spiral tightens towards the apex. This area of the shell would not be useful for creating sturdy disc beads, and indeed, the completed LSS beads from Malawi for which edge thickness could be measured (available Stage VI and Stage VII, $N=96$ ) ranged between 0.56 and $1.66 \mathrm{~mm}$, with an average thickness of $0.97 \mathrm{~mm}$. This is substantially thinner than unmodified OES available for bead manufacture, which ranges from 1.50 to $2.40 \mathrm{~mm}(N=370)$ across sub-Saharan Africa (Miller 2019: 123).

While all parts of an ostrich shell can be used to make beads, it is only the body whorl (or first whorl) of giant African land snails that would have sufficient thickness for beadmaking. This may have had unintended consequences of increasing the chances that some thinner beads would be made and also more susceptible to breakage. Indeed, broken beads from all sites (Stage VI/VII, $N=28$ ) had an average thickness of $0.90 \mathrm{~mm}$, while unbroken beads $(N=68)$ had an average thickness of $1.01 \mathrm{~mm}$. These thickness considerations combined with the prevalence of PW2 manufacture makes for some uncertainty in identifying early-stage preforms. The assemblages from all three sites contain LSS fragments that appear shaped, but in some cases, they are small or thin, so much so that it may have hindered the ability to manufacture them into beads. It is also possible that some rounded LSS discs were not intended to be beads. It is not clear if this is because they were mistakes, practice preforms (perhaps by children or inexperienced makers), or made for some other purpose, such as game tokens.

Juwayeyi (1981: 380) has suggested that preforms would not continue beyond Stage $\mathrm{V}$ if drilling produced an aperture that was considered too large. Based on data from sites in southern Malawi, he observed that "If the shell discs described below are of any help, it would seem that the shells were first randomly broken in fairly large and roughly circular pieces before perforating them and working them to a small and desirable size. After this, the edge was smoothed probably by rubbing it on a rock. It seems that the size of the perforation was the determining factor of whether one was going to end up with a bead or not. It was observed, for instance, that the perforation on discs is slightly larger than on beads. This strongly suggests that discs were discarded after an unsuccessful attempt to drill a standard shell bead size hole." Juwayeyi appears to be referring to PW2 perforated preforms as "discs," in comparison to the more finely finished "beads." This is the most comprehensive reconstruction of the chaînes opératoires of LSS beads to date, but it does not appear to be replicated in northern Malawi. Aperture measurements on eight Stage V and Stage VI preforms ranged from $0.0123\left(\mathrm{~cm}^{2}\right)$ to 0.031 . This falls well within the range of aperture sizes established for finished beads, 0.014 to 0.586 . This sample, although small, indicates that the Stage V beads recovered from HOR-1 were not discarded because of large aperture size. In fact, the apertures on these preforms fall below the average for finished beads.

Although this is the first thorough documentation of LSS manufacture in an archeological context, the dominance of 
PW2 manufacture is surprising. The ethnographic and archeological evidence for OES manufacture is overwhelmingly represented by PW1 and is believed to relate to efficiency, as PW1 permits beads to be shaped en masse. It is possible that maximizing productivity was not a consideration at these sites, that the PW2 sequence was the culturally favored method, or that the shell structure of LSS does not lend itself to shaping in the same manner. In our experiments, we found that pressure flaking LSS resulted in the propagation of cracks, some of which are visible in archeological examples (Fig. 5), and which can lead to breakage of the specimen. It would not be optimal to invest time in drilling and stringing if LSS has a higher risk of fracturing at the shaping stage. LSS also appears to be substantially thinner than OES, which may make them more susceptible to breakage during both manufacture and wear, though their microstructure provides increased durability. Only further analyses of LSS preforms from other sites will reveal whether the bead manufacturing approaches identified in the Kasitu Valley are the rule or the exception.

Our experimental data showed that LSS has very little iron in it naturally but that elevated levels can be detected with pXRF once ochre is applied. Therefore, for archeological specimens, elevated levels of iron are likely introduced onto their surfaces from either application of pigment or from adhering matrix. Readings from specimens with visible red residues will usually be lower than readings taken on archeological pigments themselves, but red residues, visible under a hand lens, are indeed a source of elevated iron readings. Furthermore, there were significant differences in iron content between unmodified archeological shells and modified shell beads and preforms from the same contexts. If iron had become elevated from exposure to the surrounding sediment, then proportions of iron should be similar between modified and unmodified specimens. The sample is not perfectly comparable, because the beads and preforms largely came from the sieved sample and are mostly $<10 \mathrm{~mm}$ in maximum dimension, whereas the unmodified shell sample was entirely from the plotted sample and should in principle all be larger than $10 \mathrm{~mm}$. However, there is no reason to believe that red coloration acquired incidentally from the sediment should differentially adhere to the surfaces of specimens smaller than $10 \mathrm{~mm}$. This analysis does not show the elemental composition of the red residues beyond the representation of iron, but our purpose was not to investigate the specific mineral status of the pigments that were applied to the shell surfaces. Instead, we offer a fast, affordable way to confirm that visible red residues are iron-rich, to quantify information that is otherwise often loosely reported as "ochre" based on visual criteria alone and potentially also to detect the presence of diffuse red pigment that is not obvious by sight.

Presentation of red, iron-rich residues on Stage III or even II preforms indicates that color was in many cases applied deliberately during production and not only incidentally acquired while being worn as a completed bead (Velliky et al. 2021). This is further supported by examples of Stage V and VI preforms/beads where the outside of the specimen contains red residue, but the aperture does not, suggesting drilling after color application. However, there are also cases where the aperture contains red residue, while the surface does not. This may be because pigment rubbed off the bead surface during wear, in which case some elevated Fe levels may still be detectable even when there are no red residues. Alternatively, for some specimens, red color may never have been applied during manufacture, but accumulated in the aperture because of a colored string, or from rubbing against a colored surface (e.g., skin or hide).

In spite of the apparent dominance of OES as a raw material, LSS beadmaking is a tradition that we now know extends at least back to the Early Holocene. Direct ages on the two preforms show similar ages ( 9000-9500 cal BP), indicating manufacture of LSS beads at different sites in the Kasitu Valley around the same time. The associated hunter-gatherer context also shows that LSS beadmaking was not a phenomenon restricted to the Iron Age, although they also occur in Iron Age deposits at KAD-1 and HOR-1. It is notable that ostriches do not currently occur in the Zambezian Phytogeographic Zone, where the open miombo woodland does not provide suitable habitat (Miller et al. 2011). Thus, use of LSS in this particular region may have been as a local substitute for OES. Future work may identify differences in local production that show different cultural traditions over time or between groups practicing different modes of subsistence, as well as trade and exchange differences between LSS and OES beads.

\section{Conclusions}

LSS beadmaking and OES beadmaking have many similarities, but their shell properties are distinct, and this requires special consideration during analysis of archeological specimens. Our study has shown that the manufacturing stages for OES can be applied to LSS, with small revisions at the beginning and end of the production sequence. Perhaps the most important distinctions between the two materials are the apparent preference for PW2 production and the natural variation in LSS thickness. Both of these make it difficult to reliably identify early-stage preforms. We propose that the minimum criteria for identifying PW2 Stage II preforms are a disc with more than $50 \%$ of the edge rounded and a minimum thickness of $\sim 0.50 \mathrm{~mm}$. Full confidence in identification is only possible after PW2 preforms have reached Stage III.

Further research is required to understand the uniqueness of LSS beadmaking traditions. LSS is an intractable material that is difficult to break, which contrasts with OES which is meant to be broken by an ostrich hatchling. Once broken into 
smaller fragments, both LSS and OES are challenging to shape into discs without further breakage. LSS beadmaking in the Kasitu Valley is dominated by PW2 manufacture, which contrasts with OES beads which are almost exclusively PW1. However, both shell types show that drilling was preferentially initiated from the inner surface, even though OES has a soft chalky mammillary layer and LSS has a slick inner nacre. Finally, the two materials do not seem to develop use wear in the same manner; in particular, it is difficult to differentiate between a LSS bead that is finished but unworn compared to a finished and well-worn bead. This suggests that the differences in raw material properties may also extend to how finished beads acquire use wear, break, and are ultimately incorporated into the archeological record at the ends of their life cycles. Our study of PW2 dominated LSS manufacture must now be compared against other LSS beadmaking assemblages from diverse regions to understand whether the Kasitu Valley beads are the norm or an outlier. Regardless of these considerations, our study shows that LSS beadmaking has been a tradition for at least the last 9500 years in southcentral Africa, filling a role that appears to have largely been dominated by OES elsewhere on the continent.

Supplementary Information The online version contains supplementary material available at https://doi.org/10.1007/s12520-021-01274-8.

Acknowledgements We thank the Malawi Department of Museums and Monuments for their logistical and permitting support, especially Dr. Elizabeth Gomani-Chindebvu, Aaron Khombe, and Chrissy Chiuma. Frederick Mapemba, Joseph Tembo, Kondwani Mwafulirwa, Henry Kalinga, Daudi Mwangomba, and the entire MALAPP crew were instrumental in recovering field and field lab data. We owe thanks to Inkosi ya Makosi M'mbelwa V, Inkosi Chindi, Inkosi Kampingo, Inkosana Nkosi, the Mzimba Heritage Association, and members of the Mzimba community near Mount Hora for their support of the project. Menno Welling and Jacob Davis provided essential logistical support. Funding for the fieldwork was provided by Wenner-Gren Foundation Grant 9432, National Geographic Society Award NGS-53412R-18, Emory University, and Yale University. Suzanne Kunitz sorted all the $3 \mathrm{~mm}$ shell from 2016 to 2018 field seasons, and Alejandra May and Kelsey Radican sorted the 2019 shell. Paul Skelley at the Florida Department of Agriculture and Consumer Services provided the sanitized modern shells used in the experimental work. Sowmya Karra and Nora Keathley at Emory University and Camilla Ledezma at Yale University conducted most of the DinoLite photography. Grace Veatch assisted with measuring shell thickness and Jacob Davis with entering data. Carla Hadden provided the text on radiocarbon dating protocols from CAIS. Andrew Zipkin provided the image of a modern giant land snail. We thank Matthew Jobbins from Nanoscience Instruments for the SEM loan and Ellery Frahm for coordinating access to it. Two anonymous reviewers provided constructive comments on our initial submission.

Availability of data and material Experimental preforms are available for study by contacting JM. Raw preform and bead count data, and photographs of each specimen, are available by contacting JCT.

Authors' contributions Conceptualization: JM and JCT; data curation: JM, JCT, HMK, and $\mathrm{CH}$; formal analysis: JM, JCT, HMK, and $\mathrm{CH}$; funding acquisition: JCT; investigation: all authors; methodology: JM,
JCT, HMK, and CH; project administration: JCT and PMK; resources: JCT; supervision: JCT and PMK; visualization: JM, JCT, CH, and HMK; writing (original draft): JM and JCT; writing (review and editing): all authors.

Funding Fieldwork funding is provided by the Wenner-Gren Foundation Grant 9432, National Geographic Society Award NGS-53412R-18, Emory University, and Yale University.

\section{Compliance with ethical standards}

Conflicts of interest/competing interests The authors declare no conflicts of interest/competing interests.

\section{Code availability N/A}

Open Access This article is licensed under a Creative Commons Attribution 4.0 International License, which permits use, sharing, adaptation, distribution and reproduction in any medium or format, as long as you give appropriate credit to the original author(s) and the source, provide a link to the Creative Commons licence, and indicate if changes were made. The images or other third party material in this article are included in the article's Creative Commons licence, unless indicated otherwise in a credit line to the material. If material is not included in the article's Creative Commons licence and your intended use is not permitted by statutory regulation or exceeds the permitted use, you will need to obtain permission directly from the copyright holder. To view a copy of this licence, visit http://creativecommons.org/licenses/by/4.0/.

\section{References}

Ademolu K, Precious O, Ebenso I, Babatunde I (2016) Morphometrics and minteral composition of shell whorls in three species of giant African snails from Abeokuta, Nigeria folia. Malacologica 24:8184. https://doi.org/10.12657/folmal.024.013

Ambrose SH (1998) Chronology of the Later Stone Age and food production in East Africa. J Archaeol Sci 25:377-392. https://doi.org/ 10.1006/jasc. 1997.0277

Arnold JE (2012) Detecting apprentices and innovators in the archaeological record: rhe shell bead-making industry of the Channel Islands. J Arch Meth Theor 19:269-305. https://doi.org/10.1007/ s10816-011-9108-1

Bleek DF (1928) The Naron: a bushman tribe of the central Kalahari. Cambridge University Press, Cambridge

Braun DR, Plummer T, Ferraro JV, Ditchfield P, Bishop LC (2009) Raw material quality and Oldowan hominin toolstone preferences: evidence from Kanjera south, Kenya. J Archaeol Sci 36:16051614. https://doi.org/10.1016/j.jas.2009.03.025

Clark JD (1956) Prehistory in Nyasaland. Nyasaland J 9:92-119

Collins B, Wojcieszak M, Nowell A, Hodgskiss T, Ames CJH (2020) Beads and bead residues as windows to past behaviours and taphonomy: a case study from Grassridge Rockshelter, Eastern Cape, South Africa. Archaeol Anthropol Sci 12:192. https://doi.org/10. 1007/s12520-020-01164-5

Dunn EJ (1931) The bushman. Bell \& Bain, Glasgow

Ecker M, Botha-Brink J, Lee-Thorp JA, Piuz A, Horwitz L (2015) Ostrich eggshell as a source of Palaeoenvironmental information in the arid interior of South Africa: a case study from Wonderwerk cave changing climates, ecosystems and environments within arid southern Africa and adjoining regions. Palaeoecol Afr 33:95115. https://doi.org/10.1201/b19410-10 
Eren MI, Lycett SJ, Roos CI, Sampson CG (2011) Toolstone constraints on knapping skill: levallois reduction with two different raw materials. J Archaeol Sci 38:2731-2739. https://doi.org/10.1016/j.jas. 2011.06.011

Eren MI, Roos CI, Story BA, von Cramon-Taubadel N, Lycett SJ (2014) The role of raw material differences in stone tool shape variation: an experimental assessment. J Archaeol Sci 49:472-487. https://doi. $\operatorname{org} / 10.1016 / j$ j.jas.2014.05.034

Frahm E, Tryon CA (2018) Origins of Epipalaeolithic obsidian artifacts from Garrod's excavations at Zarzi cave in the Zagros foothills of Iraq. J Archaeol Sci Rep 21:472-485. https://doi.org/10.1016/j. jasrep.2018.08.001

Hahn CH, Fourie L, Vedder V (1966) The native tribes of south west Africa. Frank Cass, London

Hammer Ø, Harper DAT, Ryan PD (2001) PAST: paleontological statistics software package for education and data analysis. Palaeontol Electron 4:1-9

Heckel CE (2016) Digital morphometric analysis of upper Palaeolithic beads: assessing artifact variability with user-friendly freeware. J Archaeol Sci Rep 10:893-902. https://doi.org/10.1016/j.jasrep. 2016.05.060

Hitchcock RK (2012) Ostrich eggshell jewelry manufacturing and use of ostrich products among San and Bakgalagadi in the Kalahari. Botsw Notes Rec:93-105

Hogg AG et al (2020) SHCal20 southern hemisphere calibration, 0-55, 000 years cal BP. Radiocarbon 62(4):759-778. https://doi.org/10. 1017/rdc.2020.59

Jarvis MJF, Jarvis C, Keffen RH (1985) Breeding seasons and laying patterns of the southern African ostrich Struthio camelus. Ibis 127: 442-449. https://doi.org/10.1111/j.1474-919X.1985.tb04840.x

Jordan P (2015) Technology as human social tradition: cultural transmission among hunter-gatherers. University of California Press, Berkeley

Juwayeyi Y (1981) The later prehistory of southern Malawi: a contribution to the study of technology and economy during the Later Stone Age and Iron Age Periods. University of California, Berkeley

Kandel AW, Conard NJ (2005) Production sequences of ostrich eggshell beads and settlement dynamics in the Geelbek Dunes of the Western Cape, South Africa. J Archaeol Sci 32:1711-1721. https://doi.org/ 10.1016/j.jas.2005.05.010

Langley MC (2018) Explaining the lack of emu eggshell material culture in Australia: experimental working and archaeological implications. J Archaeol Sci Rep 17:155-162. https://doi.org/10.1016/j.jasrep. 2017.11.011

Lee RB (1984) The Dobe !Kung. Holt, Rinehart and Winston, New York

Li-Chan EC, Kim H-O (2008) Structure and chemical composition of eggs. In: Egg bioscience and biotechnology. John Wiley and Sons Inc., New Jersey, pp 1-96

Marshall L (1976) The !Kung of Nyae Nyae. Harvard University Press, Cambridge

Mead AR (1961) The giant African snail: a problem in economic malacology. University of Chicago Press, Illinois

Mead AR (2004) Comparative reproductive anatomy in the South African giant land snails (Gastropoda: Pulmonata: Achatinidae). Zool Meded 78(25):417-449

Miller JM (2019) Variability in ostrich eggshell beads from the Middle and Later Stone Age of Africa. University of Alberta

Miller JM, Hallager S, Monfort SL, Newby J, Bishop K, Tidmus SA, Black P, Houston B, Matthee CA, Fleischer RC (2011) Phylogeographic analysis of nuclear and mtDNA supports subspecies designations in the ostrich (Struthio camelus). Conserv Genet 12:423-431. https://doi.org/10.1007/s10592-010-0149-x

Miller JM, Sawchuk EA, Reedman ALR, Willoughby PR (2018) Land snail shell beads in the sub-Saharan archaeological record: when, where, and why? Afr Archaeol Rev 35:347-378. https://doi.org/10. 1007/s10437-018-9305-3
Miller JM, Willoughby PR (2014) Radiometrically dated ostrich eggshell beads from the Middle and Later Stone Age of Magubike Rockshelter, southern Tanzania. J Hum Evol 74:118-122. https:// doi.org/10.1016/j.jhevol.2013.12.011

Mitchell P (2002) The archaeology of southern Africa. Cambridge Univeristy Press, Cambridge

Morris AG, Ribot I (2006) Morphometric cranial identity of prehistoric Malawians in the light of sub-Saharan African diversity. Am J Phys Anthropol 130:10-25. https://doi.org/10.1002/ajpa.20308

Nigra BT, Arnold JE (2013) Explaining the monopoly in shell-bead production on the Channel Islands: drilling experiments with four lithic raw materials. J Archaeol Sci 40:3647-3659. https://doi.org/10. 1016/j.jas.2013.04.021

Oestmo S, Marean CW (2014) Pinnacle point: excavation and survey methods. In: Smith C (ed) Encyclopedia of global archaeology. Springer New York, New York, pp 5955-5959. https://doi.org/10. 1007/978-1-4419-0465-2_1509

Orton J (2008) Later Stone Age ostrich eggshell bead manufacture in the Northern Cape, South Africa. J Archaeol Sci 35:1765-1775. https:// doi.org/10.1016/j.jas.2007.11.014

Otchoumou A, Dupont-Nivet M, Atchibri LOA, Dosso H (2010) Body proportions and chemical composition of wild and reared edible snails of Ivory Coast. Ital J Food Sci 22:105-110

Phillipson DW (1976) The prehistory of eastern Zambia. British Institute in Eastern Africa, Nairobi

Silberbauer GB (1981) Hunter and habitat in the Central Kalahari Desert. Cambridge University Press, Cambridge

Skoglund $\mathrm{P}$ et al (2017) Reconstructing prehistoric African population structure. Cell 171:59-71.e21. https://doi.org/10.1016/j.cell.2017.08.049

Smith AB, Lee RB (1997) Cho/Ana: archaeological and ethnohistorical evidence for recent hunter-gatherer/agropastoralist contact in northern Bushmanland, Namibia. S Afr Archaeol Bull 52:52-58. https:// doi.org/10.2307/3888976

Stewart BA, Zhao Y, Mitchell PJ, Dewar G, Gleason JD, Blum JD (2020) Ostrich eggshell bead strontium isotopes reveal persistent macroscale social networking across late Quaternary southern Africa. Proc Natl Acad Sci 117:6453-6462. https://doi.org/10.1073/pnas. 1921037117

Stow GW (1905) The native races of South Africa: a history of the intrusion of the Hottentots and bantu into the hunting grounds of the bushmen, the aborigines of the country. S. Sonnenschein \& Company, limited, London

Tátá F, Cascalheira J, Marreiros J, Pereira T, Bicho N (2014) Shell bead production in the Upper Paleolithic of Vale Boi (SW Portugal): an experimental perspective. J Archaeol Sci 42:29-41. https://doi.org/ 10.1016/j.jas.2013.10.029

Tryon CA (2019) The Middle/Later Stone Age transition and cultural dynamics of late Pleistocene East Africa. Evol Anthropol 28:267282. https://doi.org/10.1002/evan.21802

Van Bruggen AC (2008) New studies on the land molluscs of Malawi, a second interim progress report. Basteria 72:353-368

Van der Post L, Taylor J (1985) Testament to the bushmen. Viking Press, New York

Velliky EC, Schmidt P, Bellot-Gurlet L, Wolf S, Conard NJ (2021) Early anthropogenic use of hematite on Aurignacian ivory personal ornaments from Hohle Fels and Vogelherd caves, Germany. J Hum Evol 150:102900. https://doi.org/10.1016/j.jhevol.2020.102900

Vogel JS, Southon JR, Nelson D, Brown TA (1984) Performance of catalytically condensed carbon for use in accelerator mass spectrometry. Nucl Inst Methods Phys Res B 5:289-293. https://doi.org/10. $1016 / 0168-583 \times(84) 90529-9$

Walz J (2017) Toward an ethnoarchaeomalacology of Achatina in East Africa. Ethnobiol Lett 8:90-96. https://doi.org/10.14237/ebl.8.1. 2017.751

Ward V, Maggs T (1988) The slipped disc: a guide to the identification of shell disc-beads. Ann Natal Mus 29:407-416 
Wei Y, d'Errico F, Vanhaeren M, Peng F, Chen F, Gao X (2017) A technological and morphological study of late Paleolithic ostrich eggshell beads from Shuidonggou, North China. J Archaeol Sci 85:83-104. https://doi.org/10.1016/j.jas.2017.07.003

Werner JJ, Miller JM (2018) Distinguishing Stone Age drilling techniques on ostrich eggshell beads: an experimental approach. J Archaeol Sci Rep 22:108-114. https://doi.org/10.1016/j.jasrep. 2018.09.020

Wilmsen EN (2015) Ostrich eggshells and their beads. S Afr Archaeol Bull 70:89-105
Wingfield C (2003) Ostrich eggshell beads and the environment, past and present. In: Mitchell P, Haour A, Hobart J (eds) Researching Africa's past: new contributions from British archaeologists. Oxbow Books, Oxford, pp 54-60

Publisher's note Springer Nature remains neutral with regard to jurisdictional claims in published maps and institutional affiliations. 